\title{
CONSTRUCTION AND PROPERTIES OF SOME 6-DIMENSIONAL ALMOST COMPLEX MANIFOLDS
}

\author{
BY \\ EUGENIO CALABI $\left({ }^{(1)}\right.$
}

1. Introduction and summary. It is well known that the six-dimensional sphere $S^{6}$ admits the structure of an almost complex manifold [8; 23, pp. 209-217]. The standard way to exhibit this structure is to imbed $S^{6}$ as the unit sphere in the Euclidean 7 -space $R^{7}$ and to consider the latter as the space of "purely imaginary" Cayley numbers, then using the multiplicative properties of the Cayley algebra applied to the tangent space at each point of $S^{6}$. Ehresmann and Libermann [9] and Eckmann and Frölicher [7] showed that this particular almost complex structure is not derivable from a complex analytic one.

In the present paper we show $(\S 4)$ that the algebraic properties of the imaginary Cayley numbers induce an almost complex structure on any oriented, differentiable hypersurface $x \subset R^{7}$ just as in the case of the unit sphere. The necessary and sufficient conditions for the almost complex structure thus obtained in $X$ to be integrable (i.e. derivable from a complex analytic one) are then calculated, and one sees that no closed hypersurface can satisfy these conditions everywhere; there do exist, however, many open hypersurfaces beside the hyperplanes that satisfy the integrability conditions (Theorem 6). In $\$ 5$ we construct some special examples of hypersurfaces satisfying the integrability condition, with the additional property of being covering spaces (preserving the complex analytic structure under the covering transformations) of compact, complex manifolds. This new method of constructing compact, complex manifolds provides the first known counterexamples of the two following conjectures: (a) Are the Chern characteristic classes of a compact, complex analytic manifold uniquely determined by the real differentiable structure and the orientation? (b) If a compact, complex manifold is differentiably homeomorphic to a Kähler manifold, does it admit a Kähler metric?

The integrability condition for an almost complex manifold is given by the vanishing of a certain torsion tensor, which can be expressed, if the almost complex structure is induced by an imbedding as a hypersurface in the Cayley space, in terms of the second fundamental form. It would be interesting to

Presented to the Society, April 14, 1956 under the title Examples of 6-dimensional almostcomplex and complex manifolds and their properlies; received by the editors October 6, 1956.

(1) This paper was completed while the author had a contract with the office of Naval Research. 
investigate the integral properties of this torsion tensor for closed hypersurfaces of the Cayley space in order to find out if there are any functionals that are invariant under differentiable isotopy. The discovery of such a functional would be a first step toward finding some conditions under which a given, nonintegrable almost complex structure on a manifold is homotopic to an integrable one, an important problem, which is as tempting as it is elusive. To be more specific, it is still unknown whether the sphere $S^{6}$ or any product manifold $\mho^{2} \times S^{4}\left(\mathcal{V}^{2}=\right.$ any closed, orientable surface) admit any complex analytic structure. These manifolds admit an almost complex structure, since they can be imbedded in $R^{7}$; on the other hand we know that the almost complex structure of the product manifolds $S^{1} \times S^{5}$ and $S^{3} \times S^{3}$ induced by imbedding them in the Cayley space are homotopic to integrable ones, since the manifolds are known to admit complex analytic structures $[16 ; 2]$, and since their second homology groups are trivial, whence (cf. [8]) all almost complex structures inducing the same orientation are mutually homotopic.

2. The Cayley space. The term Cayley space denoted by $y$ is to be understood as the 7-dimensional space of Cayley numbers whose real part is zero. The algebraic structure of the Cayley algebra as an alternative division algebra over the real number field can be conveniently described in terms of invariants of the Cayley space alone; these invariants are described by operations that show the analogy of the Cayley space with the Euclidean 3-space of elementary vector analysis, where the latter is regarded as the space of purely imaginary quaternions. The notation used is an obvious adaptation of the Gibbs notation of vector analysis. Thus the Cayley space $y$ is defined for our purpose axiomatically as follows: $Y$ is a real vector space over the real number field $R$ with the two following operations (we shall denote vectors in $Y$ by capital letters and real scalars by small ones):

(a) a scalar product (also called "inner," or "dot" product) $A \cdot B$ mapping $Y \times Y$ into $R$

(b) a vector product (also called "cross" product) $A \times B$ mapping $y \times Y$ in to $Y$.

These two binary operations are subject to the following six axioms:

(1) The scalar product $A \cdot B$ is bilinear, and symmetric.

(2) The scalar product $A \cdot A$ is equivalent to a positive definite quadratic form.

(3) The vector product $A \times B$ is bilinear and skew-symmetric.

(4) The two trilinear, real valued functions $(A \times B) \cdot C$ and $A \cdot(B \times C)$ are identical, and consequently alternating; their common value is denoted hereinafter by $(A B C)$ or $(A, B, C)$, and called the triple scalar product:

$$
(A B C)=(A \times B) \cdot C=A \cdot(B \times C) .
$$

(5) The two trilinear, vector valued functions $(A \times B) \times C-(A \cdot C) B$ $+(B \cdot C) A$ and $-A \times(B \times C)+(A \cdot C) B-(A \cdot B) C$ are identical to each other, 
and consequently alternating; their common value is denoted hereinafter by $[A B C]$ or $[A, B, C]$, and called the triple vector product:

$$
\begin{aligned}
{[A B C] } & =(A \times B) \times C-(A \cdot C) B+(B \cdot C) A \\
& =-A \times(B \times C)+(A \cdot C) B-(A \cdot B) C .
\end{aligned}
$$

(6) The triple vector product (2.2) is not identically zero.

The vector space $y$ with the system of axioms just given is then uniquely determined up to an isomorphism. The structure theorem, whose proof can be found in $[11 ; 19 ; 26 ; 27]$ will be stated as follows, without proof.

THEOREM 1. A real vector space $y$ with the operation satisfying the six axioms of a Cayley space is necessarily 7-dimensional; one can select a basis $\left(I_{1}, \cdots, I_{7}\right)$ for $y$ with the two following properties:

(a) is orthonormal with respect to the inner product.

(b) The table of multiplication of the cross products $I_{j} \times I_{k}$ is the following

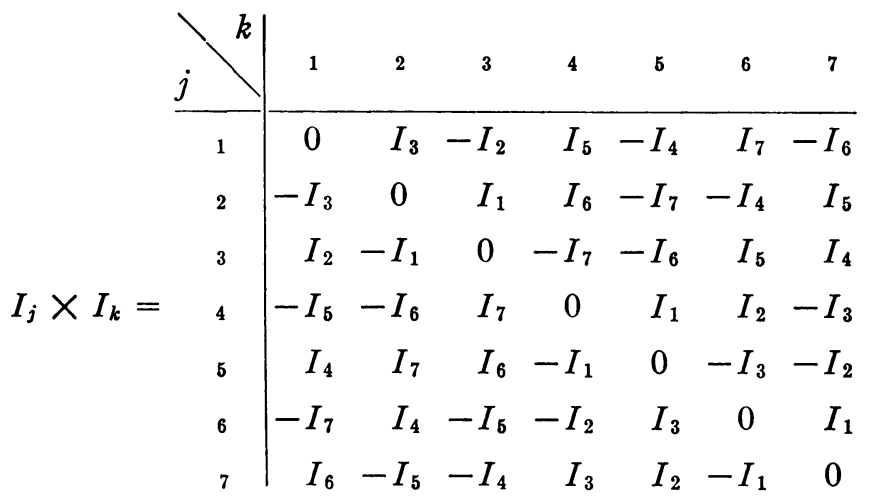

For the purpose of reference in the sequel a frame $\left(I_{j}\right)(j=1,2, \cdots, 7)$ satisfying properties (a) and (b) of Theorem 1 is called a canonical frame for Y. It can be constructed as follows: $I_{1}$ and $I_{2}$ are arbitrary, mutually orthogonal unit vectors; $I_{3}=I_{1} \times I_{2} ; I_{4}$ is an arbitrary unit vector orthogonal to $I_{1}, I_{2}, I_{3}$, and then

$$
I_{5}=I_{1} \times I_{4}, \quad I_{6}=I_{2} \times I_{4}, \quad I_{7}=-I_{3} \times I_{4} .
$$

The group of automorphisms of the Cayley space $y$ is isomorphic to the matrix group of transformations of one canonical frame into another; it is known to be the exceptional compact, simple Lie group $G_{2}$ (using E. Cartan's terminology, [3]). Of this group we need only the following structural property, also proved in [11] and elsewhere.

THEOREM 2. The subgroup $\boldsymbol{H}$ of $G_{2}$ consisting of the automorphisms of $y$ that leave the vector $I_{1}$ invariant for a fixed, canonical frame $\left(I_{j}\right)(1 \leqq j \leqq 7)$ is isomorphic to the group $S U(3)$ of unitary $3 \times 3$ matrices with unit determinant. 
To make the isomorphism explicit, let $\left(I_{j}\right)$ and $\left(I_{j}^{\prime}\right)$ be two canonical frames such that $I_{1}^{\prime}=I_{1}$. Then any vector $U=\sum_{\nu=2}^{7} a_{\nu} I_{\nu}$ orthogonal to $I_{1}$ can be represented under a mapping $\phi$ by an ordered triple $U^{\phi}$ of complex numbers

$$
U^{\phi}=\left(u_{1}, u_{2}, u_{3}\right), \text { where } u_{\alpha}=a_{2 \alpha}+i a_{2 \alpha+1} .
$$

The corresponding representation $\phi^{\prime}$ of $U$ relative to the frame $\left(I_{j}^{\prime}\right)$ is then described necessarily by a complex $3 \times 3$ matrix as follows:

$$
u_{\beta}^{\prime}=\sum_{\alpha=1}^{3} \Theta_{\beta}^{\alpha} u_{\alpha}
$$

which, as one can verify directly, is an arbitrary matrix in $S U(3)$.

3. Almost complex manifolds. Let $X=X_{(r)}^{n}$ be an $n$-dimensional, differentiable manifold of class $\mathfrak{e}^{r}(r \geqq 1)$. We shall review at first some elementary facts about abstract, differentiable manifolds, while presenting most of the notations and conventions to be used in the sequel.

A domain in $X$ will mean always a connected, open set. We say that a (real or complex valued) function $f$ in a domain $U$ of $X$ is $\mathfrak{e}^{s}$-equivalent to zero at a point $p \in U$, written $f \equiv 0 \bmod \mathfrak{A}_{(s)}(p)$, if $f$ is at least of class $\mathfrak{C}^{s}$ at $p(0 \leqq s \leqq r)$, and if for one (and hence any other) coordinate system $\left(x^{1}, \cdots, x^{n}\right)$ of class $\mathfrak{C}^{r}$ at $\boldsymbol{p}$ such that $x^{j}(\boldsymbol{p})=0(1 \leqq j \leqq n)$ we have

$$
f(\boldsymbol{q})=o\left(\sum_{j=1}^{n}\left|x^{j}(\boldsymbol{q})\right|^{s}\right) \text { as } \boldsymbol{q} \rightarrow \boldsymbol{p}, \quad \boldsymbol{q} \in U
$$

in other words $f \equiv 0$ mod $\mathfrak{A}_{(s)}(p)$, if, expressing $f$ analytically in terms of $x^{1}, \cdots, x^{n}, f$ vanishes at $p$ together with all partial derivatives of order $\leqq s$.

A tangent vector function $u$ of class $\mathfrak{C}^{8}(0 \leqq s \leqq r-1)$ in a domain $U \subset X$ is defined as a mapping which assigns to any function $f$ of class $\mathfrak{C}^{s^{\prime}}$ $\left(1 \leqq s^{\prime} \leqq r\right)$ in a subdomain $V$ of $U$ a function denoted by $u d f$ of class $\mathfrak{C}^{z^{\prime \prime}}$ $\left(s^{\prime \prime}=\min \left(s, s^{\prime}-1\right)\right)$ in $V$, called the derivative of $f$ along $u$, if the following properties are satisfied, in terms of constants $a, b$, and differentiable functions $f, g$ in $V \subset U[6$, pp. 76-79]:

(1) linearity: $u d(a f+b g)=a u d f+b u d g$;

(2) $\boldsymbol{u}$ is a derivation: $\boldsymbol{u} d(f g)=(\boldsymbol{u d f}) \cdot g+f \cdot(\boldsymbol{u d g})$;

(3) continuity: if $f \equiv 0 \bmod \mathfrak{A}_{(1)}(p)(p \in U)$, then $u d f(p)=0$.

The tangent vector functions of class $\mathfrak{e}^{s}$ in any coordinate domain $V$ in $\mathscr{X}$ (with coordinates $x^{1}, \cdots, x^{n}$, say) form a free module over the algebra of functions of class $\mathfrak{e}^{s}$ in $V$, spanned linearly by the $n$ vector functions $\boldsymbol{e}_{j}(1 \leqq j \leqq n)$ defined by

$$
\boldsymbol{e}_{j} d f=\partial f / \partial x^{j}
$$

To any function $f$ of class as least $\mathfrak{e}^{1}$ in a domain $U$ of $X$ one can naturally assign its differential $d f$, considered as a covariant vector, i.e. as a linear func- 
tional over the tangent vectors at each point of $U$ with values defined by the mapping $d f: \boldsymbol{u} \rightarrow \boldsymbol{u} d f$. The covariant vectors in any coordinate domain $V$ with coordinates $x^{1}, \cdots, x^{n}$ form an $n$-dimensional free module over the functions in $V$, dual to the module of tangent vectors, spanned by the differentials $d x^{1}, \cdots, d x^{n}$, the latter constituting the dual base to the base $\left(e_{\jmath}\right)$ of tangent vectors. Thus, if $u d x^{i}=u^{i}$, then $u=\sum_{i=1}^{n} u^{i} e_{i}$ and, for any function $f$ of class $\mathfrak{e}^{1}$

$$
u d f=\sum_{i=1}^{n} u^{i} \frac{\partial f}{\partial x^{i}} .
$$

A linear operator $\left(^{(}\right) T$ of class $\mathfrak{C}^{s}(0 \leqq s \leqq r-1)$ in a domain $U \subset X$ is an endomorphism of the space of tangent vectors at each point of $U$, such that, if $\boldsymbol{u}$ is a vector function of class $\mathcal{C}^{s^{\prime}}\left(0 \leqq s^{\prime} \leqq s\right)$ in any subdomain $V$ of $U$, then its image under $T$, written as $u T$, is a vector of class $\mathcal{e}^{s^{\prime}}$ in $V$. We regard a linear operator $T$ as synonymous with its adjoint, properly denoted by $T^{*}$, the latter defined to be the endomorphism of the module of differentials, $T^{*}: d f \rightarrow T^{*} d f$, such that

$$
T^{*} d f: u \rightarrow u\left(T^{*} d f\right)=(u T) d f .
$$

This way of writing allows us to drop the asterisk on $T^{*}$ without ambiguity: thus we may write $(u T) d f=\boldsymbol{u}(T d f)$ more simply $u T d f$. Every linear operator $T$ in this sense can be identified with the tensor of rank 2, covariant in the first argument, contravariant in the second, to which it corresponds in a natural way. In particular we denote by 1 the identity linear operator.

Definition 3.1. A space $X$ is called an almost complex manifold of class $\mathfrak{e}^{r}$, if it is a differentiable manifold (also denoted by $\mathfrak{x}$ ) of class $\mathfrak{e}^{r+1}$ with a linear operator $J$ on the tangent vectors, defined and continuous of class $\mathfrak{e}^{r}$ on the whole manifold, satisfying the operator equation

$$
J^{2}=-1
$$

An almost complex manifold is even-dimensional. In fact, if we recall that the determinant of a linear transformation is a scalar point function, and if the dimension of an almost complex manifold $X$ is $n$, we have

$$
(-1)^{n}=\operatorname{det}(-1)=\operatorname{det}\left(J^{2}\right)=(\operatorname{det}(J))^{2} \geqq 0,
$$

whence $(-1)^{n}=1$, or $n=2 m$.

By an elementary inductive process one can construct in a neighborhood of any point of an almost complex manifold $x$ a local frame (basis) for the module of tangent vectors $e_{1}, \cdots, e_{2 m}$ of class $\mathrm{e}^{r}$ (ordinarily unrelated to the frames defined in terms of the coordinates, (3.1)), such that

(2) Here and in the rest of the article all linear transformations of contravariant vectors will be considered as operations on the right; all numerical representations of contravariant vectors will be considered as one-row, many-column matrices. 


$$
\boldsymbol{e}_{j} J=(-1)^{j+1} \boldsymbol{e}_{j^{\prime}}
$$$$
\left(j^{\prime}=j-(-1)^{j} ; 1 \leqq j \leqq 2 m\right):
$$

in other words the frame $\left(\boldsymbol{e}_{j}\right)$ is characterized by the property that its image under $J$ is given respectively by $\left(\boldsymbol{e}_{2},-\boldsymbol{e}_{1}, \boldsymbol{e}_{4},-\boldsymbol{e}_{3}, \cdots, \boldsymbol{e}_{2 m},-\boldsymbol{e}_{2 m-1}\right)$ : A local frame $\left(\boldsymbol{e}_{j}\right)$ for the tangent vectors which satisfies (3.3) is then called adapted to $J$; we shall denote by $\mathcal{u}$ the aggregate consisting of any domain $U$ in an almost complex manifold $X$ of class $\mathfrak{e}^{r}$ together with a frame $\left(\boldsymbol{e}_{j}\right)$ of tangent vectors of class $\mathfrak{e}^{r}$ in $U$ adapted to $J ; \boldsymbol{u}$ is then called a local frame system.

For each local frame system $u$ we define a 1-to-1 linear representation $\psi=\psi_{\mathcal{U}}$ of the module of tangent vectors in any subdomain $V \subset U$ (and by the same token of the vector space of tangent vectors at any point $p \in U$ ) onto the $m$-dimensional module of complex-valued functions in $V$ (respectively, onto the space $C^{m}$ of ordered $m$-uples of complex numbers) by defining for any tangent vector $\boldsymbol{u}=\sum_{j=1}^{2 m} a^{j} \boldsymbol{e}_{j}$ in $V$ (resp. at $\boldsymbol{p}$ ) the $m$ components of its image $u^{\psi}=\left(u^{1}, \cdots, u^{m}\right)$ :

$$
u^{\alpha}=a^{2 \alpha-1}+i a^{2 \alpha} \quad\left(i=(-1)^{1 / 2} ; 1 \leqq \alpha \leqq m\right) .
$$

It is easy to verify that for any tangent vector $u$ (at a point in or in a subdomain of a frame system adapted to $J$ ) we have

$$
(u J)^{\psi}=i\left(u^{\psi}\right)=\left(i u^{1}, \cdots, i u^{m}\right) .
$$

Any other local frame system $\mathcal{u}^{\prime}=\left\{U^{\prime},\left(\boldsymbol{e}_{j}^{\prime}\right)\right\}$ adapted to $J$, and such that $U^{\prime}$ meets $U$, is related to $\mathcal{U}$ in $U \cap U^{\prime}$ by a linear transformation of the following type:

$$
\begin{aligned}
& \boldsymbol{e}_{2 \beta-1}^{\prime}=\sum_{\alpha=1}^{m}\left(\stackrel{\alpha}{\eta_{\beta} e_{2 \alpha-1}}+\stackrel{\alpha}{\alpha} \boldsymbol{\theta}_{2 \alpha}\right), \\
& \mathbf{e}_{23}^{\prime}=\sum_{\alpha=1}^{m}\left(-\theta_{\beta}^{\alpha} \mathbf{e}_{2 \alpha-1}+\underset{\eta_{\beta} e_{2 \alpha}}{\alpha}\right) \quad(1 \leqq \beta \leqq m),
\end{aligned}
$$

or by the easily understandable formal abbreviation:

$$
\boldsymbol{e}_{2 \beta-1}^{\prime}-i \mathbf{e}_{2 \beta}^{\prime}=\sum_{\alpha=1}^{m}\left(\eta_{\beta}^{\alpha}+i \theta_{\beta}^{\alpha}\right)\left(\boldsymbol{e}_{2 \alpha-1}-i \boldsymbol{e}_{2 \alpha}\right) \quad(1 \leqq \beta \leqq m),
$$

where the complex matrix $\Theta=\left(\eta_{\beta}^{\alpha}+i \theta_{\beta}^{\alpha}\right)$ is nonsingular and of class $\mathfrak{e}^{\text {r in }}$ $U \cap U^{\prime}$.

If the representation of the tangent vector module onto the module of ordered, complex $m$-uples corresponding to $\mathcal{u}^{\prime}$ is denoted by $\psi^{\prime}=\psi_{\mathcal{u}^{\prime}}$, then for any tangent vector $\boldsymbol{u}=\sum_{j=1}^{2 m} a^{j} \boldsymbol{e}_{j}=\sum_{j=1}^{2 m} a^{\prime j} \boldsymbol{e}_{j}^{\prime}$ in $U \cap U^{\prime}$ we have the following relation, easily derived from (3.4) and (3.6), between the two representations $u^{\psi}=\left(u^{1}, \cdots, u^{m}\right)$ and $u^{\psi^{\prime}}=\left(u^{\prime 1}, \cdots, u^{\prime m}\right)$ of $u$ :

$$
u^{\alpha}=\sum_{\beta=1}^{m} u^{\beta \beta}\left(\eta_{\beta}^{\alpha}+i \theta_{\beta}^{\alpha}\right) \quad(\alpha=1,2, \cdots, m) .
$$


Thus one concludes that the nonsingular transformation matrices connecting one representation $\psi$ of a local frame system $\mathcal{u}$ adapted to $J$ with another are precisely the nonsingular, complex $m \times m$ matrices

$$
\Theta=\left(\eta_{\beta}^{\alpha}+i \theta_{\beta}^{\alpha}\right) ;
$$

furthermore the determinant of the matrix function carrying $\left(\boldsymbol{e}_{1}, \cdots, \boldsymbol{e}_{2 m}\right)$ into $\left(\boldsymbol{e}_{1}^{\prime}, \cdots, \boldsymbol{e}_{2 m}^{\prime}\right)$ is identically equal to $|\operatorname{det}(\Theta)|^{2}$, which is positive, proving that any two frames adapted to $J$ are similarly oriented. Thus we have shown that every almost complex manifold is necessarily orientable.

Until now we have considered $J$ as an operator on tangent vectors alone. It is useful to extend the domain of action of $J$ not only to the covariant vectors, but to the whole algebra of tensor functions in $X$ as well; this is done under the following postulates:

(a) $J$ is the identity on all scalars and scalar densities.

(b) $J$ is a linear operator mapping the space of all tensors of any given type into itself.

(c) $J$ is distributive with respect to tensor multiplication.

The existence and uniqueness of the extension of $J$ from acting on contravariant and covariant vectors to arbitrary tensors is an elementary exercise: we shall only remark in this connection that $J$ is commutative with tensor transposition (permutation of factors in tensor products), and as a consequence of (3.2) is anti-commutative with simple contraction (contraction of a single contravariant argument with a covariant one): thus, if $W$ is a tensor, $P: W \rightarrow P[W]$ a simple contraction, and $J: W \rightarrow J[W]$ the action of the extended operator $J$, then $J P[W]=-P J[W]$. In particular, if we denote by $J_{1}$ the tensor representing the action of $J$ on vectors, we have the identity $J\left[J_{1}\right]=-J_{1}$.

The term "almost complex" owes its origin to the natural occurrence of an almost complex structure in every complex analytic manifold. In fact, if $X$ is a $m$-dimensional complex manifold, let $z^{1}, \cdots, z^{m}$ be a system of complex analytic (holomorphic) coordinates in a domain $V \subset x$ and let

$$
x^{2 \alpha-1}=\operatorname{Re}\left(z^{\alpha}\right) ; \quad x^{2 \alpha}=\operatorname{Im}\left(z^{\alpha}\right) \quad(\alpha=1, \cdots, m) ;
$$

then $\left(x^{1}, x^{2}, \cdots, x^{2 m}\right)$ form a system of real, analytic coordinates in $V$. We can then define $J$ in $V$ by

$$
J d x^{j}=(-1)^{j} d x^{j^{\prime}},\left(j^{\prime}=j-(-1)^{j}, j=1,2, \cdots, 2 m\right)
$$

and correspondingly for tangent vectors and tensors: then the coordinate frame of tangent vectors $\left(\boldsymbol{e}_{j}\right)$ defined by (3.1) is adapted to $J$ in $V$; in fact

$$
e_{j} J d x^{k}\left\{\begin{array}{l}
=1, \quad \text { if } j \text { is odd and } k=j+1, \\
=-1, \quad \text { if } j \text { is even and } k=j-1, \\
=0 \quad \text { in all other cases } \quad(i, k=1,2, \cdots, 2 m) .
\end{array}\right.
$$


Any other real coordinate system $\left(x^{\prime 1}, \cdots, x^{\prime 2 m}\right)$ in a domain $V^{\prime} \subset x$ meeting $V$ defines similarly by (3.1) a local frame system $\left(\boldsymbol{e}_{j}^{\prime}\right)$; the latter is adapted to $J$ in $V \cap V^{\prime}$ (since $J$ is not defined so far outside of $V$ ), if and only if $\left(x^{\prime}\right)$ $=\left(x^{\prime 1}, \cdots, x^{\prime 2 m}\right)$ in terms of $(x)$ satisfy the Cauchy-Riemann equations

$$
\frac{\partial x^{\prime 2 \beta-1}}{\partial x^{2 \alpha-1}}=\frac{\partial x^{\prime 2 \beta}}{\partial x^{2 \alpha}} ; \quad \frac{\partial x^{\prime 2 \beta-1}}{\partial x^{2 \alpha}}=-\frac{\partial x^{\prime 2 \beta}}{\partial x^{2 \alpha-1}} \quad(\alpha, \beta=1, \cdots, m),
$$

i.e. if the complex-valued coordinates $z^{\prime \beta}=x^{\prime 2 \beta-1}+i x^{\prime 2 \beta}$ are holomorphic in $V \cap V^{\prime}$. An immediate consequence of this is that, if we define $J$ in every domain of holomorphic coordinates by (3.8) and (3.9), the definition is consistent throughout $x$, so that $x$ becomes an almost complex manifold in a natural way. Equation (3.9) characterizes those real coordinate systems that are related by (3.8) to a holomorphic system, since (3.9) is equivalent to the Cauchy-Riemann equations (3.11) for a coordinate transformation. We can also combine (3.8) and (3.9) in writing the equations characterizing the holomorphic coordinates $\left(z^{1}, \cdots, z^{m}\right)$ directly:

$$
J d z^{\alpha}=i d z^{\alpha} \quad(\alpha=1, \cdots, m) ;
$$

more generally, a complex-valued function $w$ in a domain in $x$ is holomorphic, if and only if it satisfies the equation

$$
J d w=i d w,
$$

which describes the Cauchy-Riemann equations for the real and the imaginary parts of $w$ in terms of arbitrary, real coordinate systems. We shall refer to (3.12) as the generalized Cauchy-Riemann equations.

Now suppose that $X$ is a $2 m$-dimensional (in the real sense), almost complex manifold. If there exist in a neighborhood of each point $p \in X m$ solutions of (3.12), such that their differentials at $\boldsymbol{p}$ are linearly independent with respect to complex coefficients, then these $m$ functions can be taken as complex-valued coordinates in a (perhaps smaller) neighborhood of $\boldsymbol{p}$. It is readily verified that the functional transformation from one such coordinate system to another, expressed in terms of the real and imaginary parts as in (3.8) satisfies the Cauchy-Riemann equations in the usual sense (3.11): thus $x$ becomes a complex analytic manifold, whose structure is uniquely determined by the given almost complex structure. We can say then that the almost complex structure of $X$ is completely integrable to a (unique) complex analytic one. However it is known that the assumption that there exist in the neighborhood of each point $m$ solutions of (3.12) with linearly independent gradients requires some restrictive properties on $J$ in the form of integrability conditions, and it has been shown [7] that these conditions are not satisfied in certain cases. We shall derive these integrability conditions, duplicating the already published works of several authors $[7 ; 1 ; 9 ; 27]$, and in the next chapters we shall show how to construct a large class of almost complex 
manifolds, for which the integrability condition is not satisfied in most cases, and is satisfied in a few special ones; in the latter cases however the holomorphic coordinates are not given $a$ priori; their functional properties are indeed unknown to the author.

Let us assume first that the almost complex manifold $x$ is completely integrable to a complex analytic one. Let $\Phi$ be any (real- or complex-valued) function of class $\mathfrak{C}^{2}$ in a coordinate domain with holomorphic coordinates $\left(z^{1}, \cdots, z^{m}\right)$; let $\left(x^{1}, x^{2}, \cdots, x^{2 m}\right)$ be the corresponding real coordinate system defined by (3.8): we then define the Wirtinger differential operators $\partial / \partial z^{\alpha}$ and $\partial / \partial \bar{z}^{\alpha}$ formally by

$$
\begin{aligned}
& \frac{\partial}{\partial z^{\alpha}}=\frac{1}{2}\left(\frac{\partial}{\partial x^{2 \alpha-1}}-i \frac{\partial}{\partial x^{2 \alpha}}\right), \\
& \frac{\partial}{\partial \bar{z}^{\alpha}}=\frac{1}{2}\left(\frac{\partial}{\partial x^{2 \alpha-1}}+i \frac{\partial}{\partial x^{2 \alpha}}\right) .
\end{aligned}
$$

Then, using the summation convention for repeated indices (where Greek indices run from 1 to $m$ and Latin indices from 1 to $n=2 m$ ), we have the identity

$$
d \Phi=\frac{\partial \Phi}{\partial z^{\alpha}} d z^{\alpha}+\frac{\partial \Phi}{\partial \bar{z}^{\alpha}} d \bar{z}^{\alpha}
$$

and, from (3.12),

$$
J d \Phi=\frac{\partial \Phi}{\partial z^{\alpha}}\left(J d z^{\alpha}\right)+\frac{\partial \Phi}{\partial \bar{z}^{\alpha}}\left(J d \bar{z}^{\alpha}\right)=i\left(\frac{\partial \Phi}{\partial z^{\alpha}} d z^{\alpha}-\frac{\partial \Phi}{\partial \bar{z}^{\alpha}} d \bar{z}^{\alpha}\right) .
$$

Taking the exterior derivative of this last expression we have

$$
d J d \Phi=-2 i \frac{\partial^{2} \Phi}{\partial z^{\alpha} \partial \bar{z}^{\beta}} d z^{\alpha} \wedge d \bar{z}^{\beta},
$$

where we use the symbol $\wedge$ to denote exterior products; the bilinear exterior differential form $d J d \Phi$ has the property that it is equal to its transform by $J$ : in fact

$$
J d J d \Phi=-2 i \frac{\partial^{2} \Phi}{\partial z^{\alpha} \partial \bar{z}^{\beta}}\left(J d z^{\alpha}\right) \wedge\left(J d \bar{z}^{\beta}\right)=-2 i \frac{\partial^{2} \Phi}{\partial z^{\alpha} \partial \bar{z}^{\beta}} d z^{\alpha} \wedge d \bar{z}^{\beta}=d J d \Phi .
$$

Thus we obtain the following identity in a completely integrable almost complex manifold $x$ (denoting by 1 the identity operator),

$$
(1-J) d J d \Phi=0
$$

for every function $\Phi$ of class $\mathfrak{e}^{2}$.

On the other hand, if we do not assume that the almost complex manifold 
$X$ is completely integrable, we can not deduce (3.13) as an identity. In fact suppose that $\Phi$ is a function of class $\mathfrak{C}^{2}$ in a coordinate domain with coordinates $\left(x^{1}, \cdots, x^{2 m}\right)=(x)$ : the action of $J$ on the differential

$$
d \Phi=\left(\partial \Phi / \partial x^{j}\right) d x^{j}
$$

can be described in terms of the components $h_{i}^{j}(x)$ of the mixed tensor of rank 2 corresponding to $J$, as follows:

$$
J d \Phi=h_{i}^{j}(x) \frac{\partial \Phi}{\partial x^{j}} d x^{i} .
$$

Then the left-hand term of (3.13) takes the form

$$
\begin{aligned}
(1-J) d J d \Phi & =\left(\frac{\partial h_{j}^{k}}{\partial x^{i}}-\frac{\partial h_{i}^{k}}{\partial x^{j}}\right) \frac{\partial \Phi}{\partial x^{k}} \frac{d x^{i} \wedge d x^{j}}{2}-h_{i}^{p} h_{j}^{q}\left(\frac{\partial h_{q}^{k}}{\partial x^{p}}-\frac{\partial h_{p}^{k}}{\partial x^{q}}\right) \frac{\partial \Phi}{\partial x^{k}} \frac{d x^{i} \wedge d x^{j}}{2} \\
& =T_{i j}^{k}(x) \frac{\partial \Phi}{\partial x^{k}} \frac{d x^{i} \wedge d x^{j}}{2},
\end{aligned}
$$

where

$$
T_{i j}^{k}(x)=\left(\delta_{i}^{p} \delta_{j}^{q}-h_{i}^{p} h_{j}^{q}\right)\left(\frac{\partial h_{q}^{k}}{\partial x^{p}}-\frac{\partial h_{p}^{k}}{\partial x^{q}}\right) .
$$

Thus we verify that the left-hand term of (3.13) in the general case is a first order differential operator on $\Phi$, whose coefficients depend on the tensor components $h_{i}^{j}(x)$ and their first order partial derivatives; the invariance of (3.13) also shows what one can verify directly from $(3.2), h_{i}^{j}(x) h_{j}^{k}(x)=-\delta_{i}^{k}$, namely that $T_{i j}^{k}(x)$ are the components of a tensor, called the holomorphic torsion tensor of the almost complex manifold $x$. Since the left-hand side of (3.13) is a first order differential operator on $\Phi$, we have the identity for all functions $\Phi$ of class $\mathfrak{e}^{2}$

$$
(1-J) d J d \Phi=\frac{\partial \Phi}{\partial x^{k}}(1-J) d J d x^{k}
$$

In otner words as have the following useful criterion:

The equation (3.13) is identically satisfied for all functions $\phi$ of class $\mathfrak{C}^{2}$, if and only if it is satisfied for any set of functions that includes, for each point $p \in X$, a set of local coordinates at $p$.

At the same time we have also the following result:

$A$ necessary condition in order that an almost complex manifold $x$ be completely integrable to a complex analytic one is that the tensor with components $T_{i j}^{k}(x)$ vanishes identically, i.e. 


$$
T_{i j}^{k}(x)=\left(\delta_{i}^{p} \delta_{j}^{q}-h_{i}^{p} h_{j}^{q}\right)\left(\frac{\partial h_{q}^{k}}{\partial x^{p}}-\frac{\partial h_{p}^{k}}{\partial x^{q}}\right)=0 .
$$

The vanishing of the torsion tensor $T_{i j}^{k}$ is also equivalent to the complete integrability of the formal Pfaffian system (3.12). Hence, if we assume that the tensor components $h_{i}^{j}(x)$ are real analytic with respect to some coordinate system in the neighborhood of each point, then (3.14) is also sufficient for the integrability of $X$ to a complex manifold. The sufficiency theorem on the integrability condition for Pfaffian systems with analytic coefficients has been attributed at times to Frobenius; however the only references in the necessary degree of generality, that are known to the author, are in Goursat's Equations aux dérivées partielles du premier ordre (1921), where it is attributed to Bouquet and in Carathéodory's Variationsrechnung, where it is attributed to A. Mayer, both original papers having appeared in 1872. In the case at hand the theorem that (3.14) is necessary, and in the analytic case sufficient, for the integrability of $X$ to a complex manifold has been stated by Eckmann and Frölicher [7], Calabi and Spencer [1], Ehresmann and Libermann [9], and Yano [25]. The sufficiency problem in the nonanalytic case is still unsolved; however we shall not need it here, since the only cases we shall encounter where (3.14) is satisfied will be in real analytic manifolds. $\left({ }^{3}\right)$

We shall now consider a symmetric, bilinear, scalar-valued functional on the tangent vector functions, of class $\mathfrak{e}^{r}$, denoted by $Q$ and operating as follows:

$$
Q: u, v \rightarrow Q(u ; v) .
$$

It is clear that the tensorial transform $J[Q]$ of $Q$ by $J$ is again a symmetric, bilinear, scalar-valued form of class $\mathcal{C}^{r}$ : in fact we have

$$
J[Q](u ; v)=Q(u J ; v J)
$$

from the definition of the extension of $J$ to the whole tensor algebra. Furthermore $J[Q]$ is positive definite, if and only if $Q$ is positive definite: in this case the bilinear form $Q^{\prime}=Q+J[Q]$ is also positive definite and has the additional property of being invariant under $J$,

$$
J\left[Q^{\prime}\right]=Q^{\prime},
$$

since $J^{2}[Q]=Q$ identically.

Definition 3.2. A symmetric, bilinear form $Q$ in an almost complex manifold $X$ is called Hermitian with respect to $J$, if it is invariant under $J, J[Q]=Q$, i.e. if

(3) Added in proof. The proof of the sufficiency of the integrability condition (3.14) has now been established by A. Newlander and L. Nirenberg (Ann. of Math. vol. 65 (1957) pp. 391-404) without assuming analyticity. This remarkable result makes it unnecessary in the sequel to prove the analyticity of the almost complex manifolds that we shall encounter, where (3.14) is satisfied. 


$$
Q(u J ; v J)=Q(u ; v) ;
$$

it is called ANTI-HeRmitian, if $J[Q]=-Q$, i.e. if

$$
Q(u J ; v J)=-Q(u ; v)
$$

for all pairs of tangent vectors $u, v$ in $X$.

For any symmetric, bilinear form $Q$ in $x$ one can construct a new bilinear form (not necessarily symmetric) $L(\boldsymbol{u} ; \boldsymbol{v})$ by setting

$$
L(\boldsymbol{u} ; \boldsymbol{v})=Q(u J ; v) .
$$

One verifies that $Q$ is Hermitian with respect to $J$, if and only if $L$ is an alternating (i.e. exterior) form, and that $Q$ is anti-Hermitian, if and only if $L$ is symmetric. In fact the following identities hold:

$$
\begin{aligned}
L(u ; v) \pm L(v ; u) & =Q(u J ; v) \pm Q(v J ; u)=Q(u J ; v) \mp Q\left(u J^{2} ; v J\right) \\
& =(Q \mp J[Q])(u J ; v),
\end{aligned}
$$

where $Q-J[Q]$ (respectively $Q+J[Q]$ ) vanish, if and only if $Q$ is Hermitian (respectively anti-Hermitian). We shall remark in passing that, if $Q$ is Hermitian, then the alternating form $L$ is also invariant under $J$, while, if $Q$ is anti-Hermitian, then so is the symmetric form $L$.

From now on we shall assume that the almost complex manifold $X$ admits a fixed Riemannian metric, i.e. a positive definite, symmetric bilinear form $Q$ defined and of class $\mathfrak{C}^{r}$ on the whole manifold: for the existence of such a metric globally, when $X$ is paracompact, cf. Steenrod [23, p. 58]. Without loss of generality, merely by replacing $Q$, if necessary, by $(1 / 2)(Q+J[Q])$, one shows that the Riemannian metric introduced may be chosen to be Hermitian. An almost complex manifold in which a fixed, Hermitian (positive definite) metric has been incorporated into its axiomatic structure shall be called an Hermitian almost complex manifold: the scalar product of any two tangent vectors $u, v$ with respect to the metric is then abbreviated by the notation $u \cdot v$, while the alternating form defined by (3.16) from the metric is denoted by $\omega$ : thus

$$
u J \cdot v=\omega(u ; v)=-\omega(v ; u) .
$$

If we write the form $\omega$ in terms of the differentials $d x^{j}$ of the coordinates in any coordinate system $(x)=\left(x^{1}, \cdots, x^{2 m}\right)$, we have

$$
\omega=H_{j k}(x) d x^{j} d x^{k}=\sum_{1 \leq j<k \leq 2 m} H_{j k}(x) d x^{j} \wedge d x^{k} \quad\left(H_{j k}=-H_{k j}\right),
$$

where the tensor components $H_{j k}(x)$ of $\omega$ form a skew-symmetric matrix whose determinant is nowhere zero; the existence of such an exterior differential form on a differentiable (paracompact) manifold can be shown to be equivalent to the existence of an almost-complex structure [23, p. 214].

It is possible to construct inductively in a suitable neighborhood of any 
point in an Hermitian almost complex manifold $x$ a local frame system $\left(e_{j}\right)(1 \leqq j \leqq 2 m)$ of class $\mathfrak{e}^{r}$, which is at the same time adapted to $J$ (Equation (3.3)), and orthonormal with respect to the inner product,

$$
\boldsymbol{e}_{j} \cdot \boldsymbol{e}_{k}=\delta_{j k} \quad(j, k=1,2, \cdots, 2 m) ;
$$

any such frame system, i.e. satisfying both (3.3) and (3.17) is called a unitary frame with respect to $J$ and the Hermitian metric, the term being justified by the fact that under the representations $\psi$ of tangent vectors by complex $m$-uples relative to any such frame system given by (3.4) the matrices $\Theta=\left(\eta_{\beta}^{\alpha}+i \theta_{\beta}^{\alpha}\right)$ describing the transformation from one representation to another by Equations (3.7) and (3.7') are unitary.

If $\boldsymbol{u}=\left\{U ;\left(\boldsymbol{e}_{j}\right)\right\}$ is a unitary frame system and $\boldsymbol{u}=\sum_{j=1}^{2 m} a^{j} \boldsymbol{e}_{j}, \boldsymbol{v}=\sum_{j=1}^{2 m} b^{j} \boldsymbol{e}_{j}$ are any two vectors in $U$, then $\omega(\boldsymbol{u} ; \boldsymbol{v})$ is the usual symplectic product of the components of $u$ and $v$ :

$$
\omega(u ; v)=\sum_{\alpha=1}^{m}\left(a^{2 \alpha-1} b^{2 \alpha}-a^{2 \alpha} b^{2 \alpha-1}\right) .
$$

It is easy to verify in terms of local unitary frame systems that the exterior differential form $\omega^{m}$ of degree $2 m$ (i.e. the $m$-fold exterior product of $\omega$ with itself) is related to the volume element $d V$ defined by the Riemannian metric and the natural orientation defined by all frames adapted to $J$, by the identity in all positively oriented frames,

$$
\omega^{m}=m ! d V .
$$

Definition 3.3. An Hermitian almost complex manifold $x$ is called a special Hermitian (almost complex) manifold, if it is possible to cover $x$ by a family of unitary frame systems, such that the unitary matrices $\Theta=\left(\eta_{\beta}^{\alpha}+i \theta_{\beta}^{\alpha}\right)$ describing the transformation from one frame system to another by $(3.6),\left(3.6^{\prime}\right),\left(3.7^{\prime}\right)$ have unit determinant, i.e. belong to the special unitary group $S U(m)$.

It has been shown by Chern [4] that the property of an almost complex manifold $x$ admitting the additional structure of a special Hermitian manifold imposes on $x$ some topological restrictions relative to the almost complex structure already assumed: if $X$ is compact, the condition (which is both necessary and sufficient) is that the 2-dimensional cohomology class $c^{1}$ known as the first Chern class be zero. For our purpose it is sufficient to compute $c^{1}$ in the following two cases:

(a) If $x$ is a compact, complex analytic manifold and $g$ is any everywhere positive, real, scalar density of weight 1 and class $\mathfrak{e}^{2}$ (viz.: the volume element of a Riemannian metric), then the exterior differential form $\Sigma$ of degree 2 defined in each complex analytic coordinate system $(z)=\left(z^{1}, \cdots, z^{m}\right)$ by

$$
\Sigma=\frac{\partial^{2} \log g(z, \bar{z})}{\partial z^{\alpha} \partial \bar{z}^{\beta}} d z^{\alpha} \wedge d \bar{z}^{\beta}=\frac{i}{2} d J d \log g(z, \bar{z})
$$


is closed and independent of the complex coordinate system used; hence $\Sigma$ can be regarded as a closed, exterior form on all of $x$. Furthermore, if $\Sigma^{\prime}$ were the corresponding form derived from another density $g^{\prime}$, then $g^{\prime} / g$ would be a real, positive scalar defined globally in $x$, and

$$
\Sigma^{\prime}-\Sigma=\frac{i}{2} d J d \log \left(g^{\prime} / g\right)
$$

would be an exact (i.e. cohomologous to zero) exterior form: hence by de Rham's theorem (cf. [21]) $\Sigma$ defines a cohomology class [ $\Sigma$ ] of dimension 2, which is independent of the density $g$ used in (3.19). One of Chern's results is that

$$
c^{1}=-\frac{1}{2 \pi i}[\Sigma]
$$

(b) If $x$ is an algebraic manifold (over the complex field), or, more generally if $x$ is a compact, complex manifold which admits a meromorphic, scalar density $\phi$ of weight 1 (here the concept of a meromorphic density refers to a transformation rule that multiplies $\phi$ by a power of the complex analytic Jacobian of a transformation of holomorphic coordinates, and not by the real Jacobian of a coordinate transformation), then $c^{1}$ is the dual of the $(2 m-2)$ dimensional homology class of the divisor of $\phi$ (total multiplicity of manifolds of zeros minus that of poles of $\phi$ ); the invariance of this definition comes from the fact that the difference between the divisors of two densities $\phi$ and $\phi^{\prime}$ of equal weight is the divisor of a meromorphic function, $\phi / \phi^{\prime}$, which by Liouville's theorem is homologous to zero: the divisor of a meromorphic density $\phi$ is known as the canonical divisor of $x$.

Definition 3.4. A manifold $X$ is called Kählerian, if it is an Hermitian almost complex manifold, and if the covariant derivative of the tensor $J$ (representing $J$ ) with respect to the Riemannian metric connection vanishes, or, equivalently, if the covariant derivative of $\omega$ vanishes.

It is known (cf. $[1 ; 17]$ ) and can be verified by direct computation that a necessary and sufficient condition for an Hermitian almost complex manifold $x$ to be Kählerian is that, first of all the integrability condition (3.14) in terms of $J$ be satisfied, and secondly that the exterior differential form $\omega$ be closed. The necessity of the two conditions is immediate, since, if $x$ is quasi-Kählerian, taking for any point $p \in X$ a system of geodesic coordinates at $p$, all the first partial derivatives of the tensor components $h_{j}^{k}$ of $J$ and $H_{j k}$ of $\omega$ vanish at $p$. The sufficiency is proved by a similar argument.

Definition 3.5. An Hermitian almost complex manifold $x$ is called Hermitian symplectic, if the exterior form $\omega$ determined by $J$ and the metric is closed.

It has been shown (cf. [17] also for earlier references) that the existence of a symplectic structure on a compact manifold imposes topological restrictions beyond those required for an almost complex structure; however not all 
the topological properties that Hodge [14] proved for Kähler manifolds have been shown to hold for symplectic manifolds: it would seem indeed surprising if this were the case; nevertheless there is no example known to the author of a compact, symplectic manifold that is not known $a$ priori to admit a Kählerian structure.

Given an Hermitian almost complex manifold $x$, we shall consider, beside the usual inner product $\boldsymbol{u} \cdot \boldsymbol{v}$ of two tangent vectors, also their Hermitian product

$$
(\boldsymbol{u}, \boldsymbol{v})=\boldsymbol{u} \cdot \boldsymbol{v}-i \omega(\boldsymbol{u} ; \boldsymbol{v})
$$

which takes the form, relative to the representation $\psi$ given by (3.4) relative to any local unitary frame system, letting $u^{\psi}=\left(u^{1}, \cdots, u^{m}\right), v^{\psi}=\left(v^{1}, \cdots\right.$, $\left.v^{m}\right)$,

$$
(u, v)=\sum_{\alpha=1}^{m} u^{\alpha} \bar{v}^{\alpha},
$$

which is the canonical form for the Hermitian product. The Hermitian product thus has the following properties:

$$
\begin{aligned}
& \text { (a) }(u, v)=(v, u)^{-} ; \\
& \text {(b) }(u J, v)=-(u, v J)=i(u, v) ; \\
& \text { (c) }(u, v)+(v, u)=2 u \cdot v ; \\
& \text { (d) }(u, v)-(v, u)=-2 i \omega(u ; v) \text {. }
\end{aligned}
$$

The final topic in this general review of almost complex manifolds concerns the relationships of linear operators $T$ on the space of tangent vectors at each point with the structural operator $J$ and with the metric, if the manifold is also Hermitian.

Definition 3.6. A linear operator $T$ operating pointwise on the space of tangent vectors in a Riemannian manifold $X$ is called symmetric with respect to the Riemann metric, if for every pair of tangent vectors $u, v$ it satisfies

$$
u T \cdot v=u \cdot v T
$$

if $X$ is almost complex, then $T$ is called complex-linear (respectively: complexantilinear), if

$$
T J=J T \quad(\text { respectively: } T J=-J T) ;
$$

if $X$ is Hermitian almost complex, then $T$ is called an Hermitian operator, if it is both complex-linear with respect to $J$ and symmetric, i.e. if

$$
(u T, v)=(u, v T) .
$$

Given any symmetric transformation $T$ in an Hermitian almost complex manifold $X$ there exists at each point $p \in X$ an orthonormal frame $\left(\boldsymbol{e}_{j}\right)$ 
$(j=1,2, \cdots, 2 m)$, ordinarily unrelated to $J$, such that each $e_{j}$ is a characteristic vector, i.e. satisfies

$$
\boldsymbol{e}_{j} T=\lambda_{j} \boldsymbol{e}_{j} \quad(1 \leqq j \leqq 2 m),
$$

and the corresponding proper value $\lambda_{j}$ is real; conversely, if all the proper values of $T$ at each point are real and if their corresponding characteristic vectors can be chosen mutually orthogonal, then $T$ is symmetric. If $T$ in addition is Hermitian, then in particular it commutes with $J$; consequently it is possible to choose at each point a unitary frame $\left(e_{j}\right)$ satisfying (3.27) with $\lambda_{2 \alpha}=\lambda_{2 \alpha-1}=\mu_{\alpha}$, i.e.

$$
\boldsymbol{e}_{2 \alpha-1} T=\mu_{\alpha} \mathbf{e}_{2 \alpha-1} ; \quad \boldsymbol{e}_{2 \alpha} T=\mu_{\alpha} \boldsymbol{e}_{2 \alpha} \quad(\alpha=1,2, \cdots, m) .
$$

If the proper values of a symmetric operator $T$ are all positive, we say that $T$ is positive definite; this property is equivalent to the inequality

$$
u T \cdot u>0
$$

for all tangent vectors $u \neq 0$.

If $S$ is an orthogonal linear operator on the tangent vectors, i.e. if it satisfies the identity

$$
u S \cdot v S=u \cdot v
$$

for all vectors $u$ and $v$, and if $T$ is symmetric, then $S T S^{-1}$ is also symmetric, and similar to $T$, that is to say, it has the same proper values as $T$ including multiplicity; in particular, if $T$ is positive definite, then so is $S T S^{-1}$; in this last case the operator

$$
T+S T S^{-1}
$$

is also positive definite, because of (3.29). We shall consider the special case where $S=J$, the latter being clearly orthogonal (equivalent to saying that the metric is Hermitian); because of (3.2) we have $J^{-1}=-J$. For any symmetric $T$ the operator $J T J^{-1}=-J T J$ is symmetric and similar to $T$; furthermore the operator $T^{\prime}$ defined by

$$
T^{\prime}=T+J T J^{-1}=T-J T J
$$

is obviously Hermitian; if $T$ is positive definite, then so is $T^{\prime}$. Conversely, if $T^{\prime}=0$, then $T$ can not be positive definite; indeed the trace $\operatorname{Sp}(T)$ (sum of its proper values, or contraction) of $T$ vanishes, since $\operatorname{Sp}(T)=\operatorname{Sp}\left(J T J^{-1}\right)$ $=-\mathrm{Sp}(J T J)$ and hence

$$
\mathrm{Sp}(T)=\frac{1}{2} \mathrm{Sp}(T-J T J)=\frac{1}{2} \mathrm{Sp}\left(T^{\prime}\right)=0 .
$$

4. Hypersurfaces in the Cayley space. Let $X$ be a 6-dimensional, orientable, differentiable manifold and let $F$ be a regular, differentiable mapping of $X$ into the Cayley space $\mathcal{Y}$, whose structure is discussed in $\S 2$. By a regular 
mapping $F$ we mean a differentiable mapping of class $\mathcal{C}^{2}$, such that the $6 \times 7$ matrix of functions representing the first partial derivatives in any parametric representation of $F$ has rank 6 at every point of $x$ : in other words $F$ is locally a differentiable imbedding; no assumption will be made about the behavior of $F$ in the large, so that we admit self-intersections and self-overlaps of $X$ under $F$, as well as the possibility of discrete sets of points in $X$ having images in $Y$ accumulating at points (that may or may not belong to $F(X)$ ).

Our differential-geometric study of $X$ will be based on the invariants induced by $F$ from the invariants defined in $\mathcal{Y}$ by the group $G_{2}$ extended by the group of translations. The Cayley space $y$ is homogeneous under this transformation group and, since $G_{2} \subset S O(7)$ (the group of rotations in Euclidean 7 -space), the invariants to be considered will include those of classical differential geometry.

In analogy with the notation used in the last chapter, in which $d f$, the differential of a scalar function $f$ in $X$, is linear, scalar-valued functional on the tangent vectors $u$ at each point $p \in X$, we regard $X=F(\boldsymbol{p})$ as the image under $F$ of a variable point $\boldsymbol{p}$, and $d X(\boldsymbol{p})$ as the linear functional on tangent vectors $\boldsymbol{u}$ at $\boldsymbol{p}$, whose value $\boldsymbol{u} d X$ is the derivative of $X$ along $\boldsymbol{u}$ at $\boldsymbol{p}$ : hence $d X$ is a $Y$-valued linear functional on the vectors $u$, and $u d X$ can then be regarded as the image of $\boldsymbol{u}$ induced by $F$, i.e. as the vector in $\mathcal{Y}$ tangent to $F(X)$ at $F(\boldsymbol{p})$ corresponding to $u$ for each $\boldsymbol{p} \in X$ where $\boldsymbol{u}$ is defined. The condition that $F$ is regular can be formulated as saying that for each $p \in X$ the linear mapping $d X(\boldsymbol{p})$ is a monomorphism (isomorphism into) of the tangent space of $X$ at $p$ into $Y$. Since the vectors $u d X$ thus obtained are invariant under the translations of $F$ in $Y$, we can apply to them all the operations that represent the invariants of $G_{2}$, such as the scalar and vector products already discussed in Chapters 1 and 2.

As in classical differential geometry the mapping $F$ induces a quadratic form, the first fundamental form

$$
d s^{2}=d X \cdot d X
$$

which is a positive definite quadratic form on the tangent vectors: the corresponding Riemannian inner product between tangent vectors $u$ and $v$ is

$$
\boldsymbol{u} \cdot \boldsymbol{v}=\mathbf{u} d X \cdot \mathbf{v} d X .
$$

Since the image under $d X(p)$ of the tangent vectors at each $p \in X$ forms a hyperplane in $\mathcal{Y}$, one can choose either one of two opposite unit vectors orthogonal to the tangent space image. We recall that $y$ has a natural orientation given by a canonical frame $\left(I_{j}\right)(j=1, \cdots, 7)$, since the group $G_{2}$ preserves orientation (Corollary 1 , Theorem $2, \S 2$ ); since $X$ is orientable, to either orientation we choose for $X$ we shall choose the unit normal vector $N=N(\boldsymbol{p})$ at $F(\boldsymbol{p})$ such that, if $\left(\boldsymbol{e}_{1}, \cdots, \boldsymbol{e}_{6}\right)$ is a positively oriented frame of tangent vectors at $\boldsymbol{p}$, then the frame $\left(N(\boldsymbol{p}), \boldsymbol{e}_{1} d X, \cdots, \boldsymbol{e}_{6} d X\right)$ at $F(\boldsymbol{p})$ is 
positively oriented relative to a canonical frame for $\mathcal{Y}$. Opposite orientations of $x$ determine opposite choices of $N$. If $F$ is of class $\mathbb{C}^{r}$, then $N(\boldsymbol{p})$ is of class $\mathrm{e}^{r-1}$, and if $Y=Y(\boldsymbol{p})$ is a $\mathcal{Y}$-valued, translation invariant vector function of $p$ of class $\mathfrak{C}^{\rho}, 0 \leqq \rho<r$, such that $N \cdot Y$ vanishes identically, then there exists a uniquely determined vector function $\boldsymbol{u}=\boldsymbol{u}(\boldsymbol{p})$, of class $\mathfrak{e}^{\rho}$, such that $\boldsymbol{u} d X$ $=Y$.

The second fundamental form is defined as the component along $N$ of the second order differential $D^{2} X$ : it is also written as a symmetric, bilinear form on the tangent vectors in $X$ whose value is a scalar of odd kind (by this we mean that if the orientation of $X$ is reversed, the value is replaced by its negative), denoted by $\left(N \cdot D^{2} X\right)(u ; v)$. We compute it formally as follows: consider the identities

$$
N \cdot \mathbf{u} d X=0
$$

and

$$
N \cdot N=1
$$

differentiating absolutely (i.e. regarding $\boldsymbol{u}$ formally as a constant) these two identities along a second vector $\boldsymbol{v}$ we obtain

$$
\left(N \cdot D^{2} X\right)(v ; u)+v d N \cdot u d X=0
$$

and

$$
2 N \cdot v d N=0 .
$$

The last of these identities implies that for each $v$ there exists a tangent vector in $x$, which depends linearly on $v$, and hence a linear operator $K: v \rightarrow v K$, such that

$$
v d N=-v K d X .
$$

From $(4.5)$ and the identity $\left(N \cdot D^{2} X\right)(\boldsymbol{v} ; \boldsymbol{u})=\left(N \cdot D^{2} X\right)(\boldsymbol{u} ; \boldsymbol{v})$ we obtain (cf. Definition 3.6)

$$
\text { (4.8) } u K \cdot v=-u d N \cdot v d X=\left(N \cdot D^{2} X\right)(u ; v)=\left(N \cdot D^{2} X\right)(v ; u)=v K \cdot u \text {, }
$$

which shows that $K$ is the operator corresponding to the second fundamental form (Weingarten's equations) and that it is symmetric with respect to the Riemannian structure defined by the first fundamental form. The proper values $\kappa_{1}, \cdots, \kappa_{6}$ of $K$ are called the principal curvatures of $X$ at each point $p$ and the corresponding normalized characteristic vectors $e_{1}^{\prime}, \cdots$, $\mathbf{e}_{6}^{\prime}$ are the respective principal directions. Thus

$$
\boldsymbol{e}_{j}^{\prime} K=\kappa_{j} \boldsymbol{e}_{j}^{\prime} \quad(j=1, \cdots, 6) .
$$

In the sequel we shall deal chiefly with the mean curvature of $x$, defined as usual by 


$$
H=\frac{1}{6} \sum_{j=1}^{6} \kappa_{j}=\frac{1}{6} \operatorname{Sp}(K) .
$$

We shall now recall two theorems on hypersurfaces in Euclidean space.

THEOREM A. If a hypersurface $x$ in Euclidean space is of class $\mathfrak{C}^{2}$, and if its mean curvature $H$ vanishes identically, then $X$ is a real analytic hypersurface. The condition $H=0$ means that $X$ is locally a solution of the Plateau problem.

The proof of the first part of this statement is contained in [20], [18], and [15], which deal with the differentiability of solutions of nonlinear, second order, elliptic partial differential equations. A proof of the second half of the statement can be found, for instance, in Eisenhart's textbook [10, pp. 176179].

Theorem B. If $\mathfrak{X}$ is a closed hypersurface of class $\mathfrak{e}^{2}$ in Euclidean space, then there exists a nonempty region $U \subset x$, where the second fundamental form is definite (positive or negative, depending on the orientation), i.e. where all the principal curvatures $\kappa_{j}$ are $\neq 0$ and have equal sign.

This proposition, known as Hadamard's lemma, is proved for instance in [5, p. 23], by taking an arbitrary point $A$ in the Euclidean space and letting $U$ be a sufficiently small neighborhood relative to $X$ of the point $p \in X$ whose Euclidean distance from $A$ is a maximum.

Thus far we have confined our discussion of the regular mapping $F$ of the orientable manifold $x$ into the Cayley space $Y$ exclusively to the invariants of the Euclidean structure of $\mathcal{Y}$, i.e. making use only of the scalar product and orientation of $\mathcal{Y}$, and not of the properties of the vector product. We shall now take the latter into consideration.

Let $p \in \mathscr{X}$, let $X=F(p)$ be its image in $Y$, let $\boldsymbol{u}=\boldsymbol{u}(\boldsymbol{p})$ any tangent vector at $\boldsymbol{p}$, and let $N=N(\boldsymbol{p})$ the normal vector to $F(\mathscr{X})$ at $F(\boldsymbol{p})$ : then the vector

$$
N(\boldsymbol{p}) \times \mathbf{u} d X(\boldsymbol{p})
$$

is tangent to $F(x)$ at $F(\boldsymbol{p})$, since $N \cdot(N \times u d X)=(N, N, u d X)=0$; hence there exists a unique tangent vector $v$ at $p$ such that

$$
v d X=N \times u d X \text {. }
$$

Since the mapping $u \rightarrow v$ is obviously linear, we can denote it, as in the beginning of $\S 3$, by the letter $J$ : thus $J$ is defined by

$$
u J d X=N \times u d X .
$$

It follows then from (2.2), (4.3), and (4.4) that

$$
\begin{aligned}
u J^{2} d X & =N \times(N \times \mathbf{u} d X) \\
& =-[N, N, \boldsymbol{u} d X]+(N \cdot \mathbf{u} d X) N-(N \cdot N) \mathbf{u} d X=-\mathbf{u} d X
\end{aligned}
$$


thus $J$ satisfies Equation (3.2) characterizing the structural operator of an almost complex manifold. Since the immersion $F$ of $x$ into $y$ is assumed to be of class $\mathfrak{C}^{2}$, it follows that $J$ is of class $\mathfrak{C}^{1}$. From (2.1) and (4.11) we have the further identity

$$
\begin{aligned}
u J \cdot v J & =(u J d X) \cdot(v J d X)=(N \times u d X) \cdot(N \times v d X) \\
& =\{(N \times u d X) \times N\} \cdot v d X=\{-N \times(N \times u d X)\} \cdot v d X \\
& =u d X \cdot v d X=u \cdot v .
\end{aligned}
$$

The last three equations imply the following important result.

Theorem 3. A regular mapping $F$ of an oriented, 6-dimensional manifold $X$, of class $\mathfrak{C}^{\rho}(\rho \geqq 1)$ into the Cayley space $\mathcal{Y}$ induces in $X$ the structure of an Hermitian, almost complex manifold under the linear operator $J$ defined by (4.10) and the induced Riemannian metric.

The exterior form of degree $2 \omega(\boldsymbol{u} ; \boldsymbol{v})$ defined as in (3.16) from $J$ and the metric can be written as follows:

$$
\omega(\boldsymbol{u} ; \boldsymbol{v})=\mathbf{u} J \cdot \boldsymbol{v}=(N, \boldsymbol{u} d X, \boldsymbol{v} d X) .
$$

We shall now follow the procedure outlined in $\$ 3$ to find the necessary and sufficient conditions on $F$ in order that the induced almost complex structure on $x$ be completely integrable, or respectively that the exterior form $\omega$ define a symplectic structure in $x$.

Theorem 4. A 6-dimensional, oriented manifold $x$ with the almost complex structure induced on it by a regular mapping $F$ of class $\mathfrak{e}^{2}$ of $x$ into the Cayley space $Y$ is integrable to a complex analytic manifold, if and only if the linear operator $K$ on its tangent space defined by the second fundamental form satisfies everywhere on $x$ the equation

$$
K-J K J=0 .
$$

Comparing Equation (4.14) with Definition 3.2, one can rephrase the necessary and sufficient condition that the almost complex structure induced on $X$ by $F$ be completely integrable is that the second fundamental form be anti-Hermitian with respect to $J$, or equivalently that the linear operator $K$ associated with the second fundamental form be complex-antilinear (cf. Definition 3.6).

Proof. From Equations (3.13) and (3.13') one sees that the integrability condition in the case at hand is equivalent to the equation

$$
(1-J) d J d X=0,
$$

where the left-hand member is an exterior, bilinear, differential form at each point $\boldsymbol{p} \in \mathfrak{X}$, with values in $\mathcal{Y}$, tangent to $F(\mathfrak{X})$ at $X=F(\boldsymbol{p})$. We shall compute this left-hand member in terms of the basic invariants of $y$. 
The vector-valued differential $J d X$ maps a tangent vector $\boldsymbol{u}$ into

$$
u J d X=N \times u d X
$$

hence its exterior derivative $d J d X$ maps any two tangent vectors $u, v$ into $(u ; v) d J d X=u d N \times v d X-v d N \times u d X=-u K d X \times v d X-u d X \times v K d X$.

It is convenient to split this last expression into the components respectively tangent to $F(\mathscr{X})$ and parallel to $N$, according to the formula $A=N \times(A \times N)$ $+(N \cdot A) N$ for $A \in Y$ : thus, applying the identities of Chapter 1 and Equation (4.3), we have

$$
\begin{aligned}
(\boldsymbol{u} ; \boldsymbol{v}) d J d X= & N \times\{N \times(\mathbf{u} K d X \times \mathbf{v} d X)+N \times(\mathbf{u} d X \times v K d X)\} \\
& -\{(N, \mathbf{u} K d X, \boldsymbol{v} d X)+(N, \mathbf{u} d X, \boldsymbol{v} K d X)\} N \\
= & N \times\{-[N, \mathbf{u} K d X, \boldsymbol{v} d X]-[N, \mathbf{u} d X, \boldsymbol{v} K d X]\} \\
& -\{(N, \mathbf{u} K d X, \boldsymbol{v} d X)+(N, \mathbf{u} d X, \boldsymbol{v} K d X)\} N .
\end{aligned}
$$

Similarly, denoting by $K^{\prime}$ the linear operator $J K J^{-1}=-J K J$, we evaluate the vectorial exterior form $J d J d X$ as follows:

$$
\begin{aligned}
(u ; v) J d J d X= & -u J K d X \times v J d X-u J d X \times v J K d X \\
= & -\left(N \times u K^{\prime} d X\right) \times(N \times v d X)-(N \times u d X) \times\left(N \times v K^{\prime} d X\right) \\
= & N \times\left\{\left[N, u K^{\prime} d X, v d X\right]+\left[N, u d X, v K^{\prime} d X\right]\right\} \\
& -\left\{\left(N, u K^{\prime} d X, v d X\right)+\left(N, u d X, v K^{\prime} d X\right)\right\} N .
\end{aligned}
$$

The normal components of the latter expression satisfy the identities $\left(N, u K^{\prime} d X, v d X\right)$

$$
\begin{aligned}
& =\left(N \times u J K J^{-1} d X\right) \cdot v d X=u J K d X \cdot v d X=u J K \cdot v=u J \cdot v K \\
& =u J d X \cdot v K d X=(N \times u d X) \cdot v K d X=(N, u d X, v K d X)
\end{aligned}
$$

and similarly

$$
\left(N, u d X, v K^{\prime} d X\right)=(N, u K d X, v d X) .
$$

Thus, subtracting equation (4.17) from (4.16), one verifies that the normal components cancel each other, leaving only a tangential term, whose vanish. ing is equivalent to (4.15):

$$
\begin{aligned}
& (u ; v)(1-J) d J d X \\
& \quad=-N \times\left\{\left[N, u\left(K+K^{\prime}\right) d X, v d X\right]+\left[N, u d X, v\left(K+K^{\prime}\right) d X\right]\right\}
\end{aligned}
$$

In order to prove the assertion we must show that the right hand member of (4.18) vanishes, if and only if $K+K^{\prime}$ itself is zero. Since $K$ is a symmetric operator (Equation (4.8) and Definition 3.6), it follows that $K+K^{\prime}=K-J K J$ $=K+J K J^{-1}$ is Hermitian (cf. Equation (3.30)): hence one can choose at 
each point $p \in X$ a unitary frame $e_{1}, \cdots, e_{6}$ of tangent vectors that are characteristic vectors of $K+K^{\prime}$ corresponding to three real proper values $\mu_{1}, \mu_{2}, \mu_{3}$, i.e. such that

$$
\mathbf{e}_{2 \alpha-1}\left(K+K^{\prime}\right)=\mu_{\alpha} \mathbf{e}_{2 \alpha-1} ; \quad \mathbf{e}_{2 \alpha}\left(K+K^{\prime}\right)=\mu_{\alpha} \mathbf{e}_{2 \alpha} \quad(\alpha=1,2,3) .
$$

Consider now a canonical frame $I_{1}, \cdots, I_{7}$ in $\mathcal{Y}$ at $F(p)$ such that $I_{1}=N$ and the remaining ones are tangent to $F(\mathscr{X}): I_{j}=\boldsymbol{e}_{j-1}^{\prime} d X \quad(2 \leqq j \leqq 7)$, where $\left(e_{1}^{\prime}, \cdots, e_{6}^{\prime}\right)$ is a unitary frame at $p$, as one readily verifies from Theorems 1 and 2 . Then the frame $\left(\boldsymbol{e}_{j}\right)$ of characteristic vectors of $K+K^{\prime}$ is related to the frame $\left(\boldsymbol{e}_{j}^{\prime}\right)$ as in $\left(3.6^{\prime}\right)$ by a unitary $3 \times 3$ matrix $\Theta=\left(\eta_{\beta}^{\alpha}+i \theta_{\beta}^{\alpha}\right)$; since it is obviously possible to replace $e_{1}$ and $e_{2}$ without loss of generality by $e_{1} \cos \tau$ $+e_{2} \sin \tau$ and $-e_{1} \sin \tau+e_{2} \cos \tau$ respectively, where $\tau$ is any real number, one can choose $\tau$ so that the matrix $\Theta$ becomes unimodular. By Theorem 2 the frame $\left(\boldsymbol{e}_{j}\right)$ thus adjusted has the property that the 7 -frame $\left(N, \mathbf{e}_{1} d X, \cdots\right.$, $\left.e_{6} d X\right)$ at $F(p)$ in $y$ is canonical in the sense of Theorem 1 ; consequently from (2.3) we have the identities

$$
\left[N, e_{2 \alpha} d X, e_{2 \beta} d X\right]=e_{2 \gamma} d X(\alpha, \beta, \gamma=\text { any cyclic permutation of } 1,2,3),
$$

whence from (4.18) and (4.19) we have, by replacing $u$ and $v$ by $e_{2 \alpha}$ and $e_{2 \beta}$ respectively,

$$
\begin{aligned}
\left(\boldsymbol{e}_{2 \alpha} ; \mathbf{e}_{2 \beta}\right)(1-J) d J d X & =-\left(\mu_{\alpha}+\mu_{\beta}\right) N \times\left[N, \mathbf{e}_{2 \alpha} d X, \mathbf{e}_{2 \beta} d X\right] \\
& =\left(\mu_{\alpha}+\mu_{\beta}\right) e_{2 \gamma-1} d X,
\end{aligned}
$$

where $\alpha, \beta, \gamma$ are cyclic permutations of $1,2,3$. Thus a necessary condition for the integrability condition (4.15) is that $\mu_{\alpha}+\mu_{\beta}=0$ for $\alpha, \beta$ ranging over distinct pairs of values $1,2,3$; this clearly implies that each $\mu_{\alpha}=0$, and hence that $K+K^{\prime}=0$, or equation (4.14); the latter is clearly sufficient for the vanishing of both members of (4.18) identically in $u$ and $v$. Thus we have just proved that the integrability condition (3.13) for the almost complex structure induced on $X$ by $F$ is Equation (4.14).

In order to prove that in our case the integrability condition (3.13) is sufficient, it is sufficient to notice that, if $K+K^{\prime}=0$, it follows from equation (3.31) that the trace $\operatorname{Sp}(K)$ vanishes identically, and hence that $X$ is mapped (locally) into a minimal hypersurface. Since the mapping $F$ can be defined by functions satisfying second order elliptic equations with analytic coefficients, as we recalled in Theorem A earlier in this chapter, we deduce that $F(x)$ is locally an analytic hypersurface, and in this case the sufficiency of the integrability condition (3.13) has been already established. This completes the proof of Theorem 4.

Corollary. If $\Upsilon$ is a compact, orientable, 6-dimensional manifold, and $F$ a regular mapping of $X$ into $\mathcal{Y}$, then there exists a nonempty domain $U \subset X$ such that there are no solutions of the generalized Cauchy-Riemann equations (relative 
to the induced almost complex structure) in any subdomain of $U$ except constants.

Proof. It follows from Hadamard's lemma (quoted earlier in this chapter as Theorem $B$ ) that, if $X$ is compact, then there exists a point in a neighborhood of which the second fundamental form is either positive or negative definite, depending on the orientation. Assuming as we well may that it is positive definite, in that domain the operator $K+K^{\prime}$ is a positive definite Hermitian operator, as we verified at the end of $\$ 3$, and hence the proper values $\mu_{1}, \mu_{2}, \mu_{3}$ of $K+K^{\prime}$ are all positive in some region $U$. Let $w$ be any holomorphic function in a subdomain $V$ of $U$, let $p$ be any point in $V$, and let $\left(\boldsymbol{e}_{j}\right)(1 \leqq j \leqq 6)$ be a unitary frame at $\boldsymbol{p}$ consisting of characteristic vectors of $K+K^{\prime}$, adjusted so that $\left(N, e_{1} d X, \cdots, e_{6} d X\right)$ is a canonical frame for $Y$ at $F(p)$. Then on the one hand, since $w$ is holomorphic, we have $d w=-i J d w$, and hence

$$
(1-J) d J d w=0 ;
$$

on the other hand we have from Equation (4.20)

$$
\left(\boldsymbol{e}_{2 \alpha} ; \boldsymbol{e}_{2 \beta}\right)(1-J) d J d w=\left(\mu_{\alpha}+\mu_{\beta}\right) \boldsymbol{e}_{2 \gamma-1} d w,
$$

and similarly

$$
\left(\boldsymbol{e}_{2 \alpha-1} ; \boldsymbol{e}_{2 \beta-1}\right)(1-J) d J d w=\left(\mu_{\alpha}+\mu_{\beta}\right) \mathbf{e}_{2 \gamma} d w,
$$

where $\alpha, \beta, \gamma$ are any cyclic permutation of $1,2,3$ : since the $\mu_{\alpha}$ 's are all positive at $\boldsymbol{p}$, it follows from the last three equations that $d w$ vanishes at $p$, and hence everywhere in $V$. Thus we have proved that, if $w$ is holomorphic in $V$, it is a constant.

A further analysis of the result just established reveals that, if $F$ is an analytic mapping of a compact $X$ into $\mathcal{Y}$, then there are no nonconstant holomorphic functions anywhere in $x$.

We pass now to the second problem, namely that of deriving the conditions on the mapping $F$ in order that it induce on $X$ a symplectic structure in terms of the exterior form $\omega$ defined by (4.13).

Theorem 5. A 6-dimensional, oriented, differentiable manifold $x$ is a symplectic manifold with respect to the Hermitian, almost complex structure induced by a regular, differentiable mapping $F$ of $X$ into $Y$, if and only if the linear operator $K$ defined by its second fundamental form satisfies the two equations

$$
K+J K J=0 ; \quad \operatorname{Sp}(K)=6 H=0,
$$

where $H$ is the mean curvature of $F(x)$.

Proof. From formula (4.13) expressing $\omega(\boldsymbol{u} ; \boldsymbol{v})$ as a triple scalar product of vectors in $\mathcal{Y}$ we obtain the formula for the exterior derivative $d \omega$ as follows: 


$$
\begin{aligned}
& d \omega(u ; v ; w) \\
(4.22) & =(u d N, v d X, w d X)+(u d X, v d N, w d X)+(u d X, v d X, w d N) \\
& =-(u K d X, v d X, w d X)-(u d X, v K d X, w d X)-(u d X, v d X, w K d X) .
\end{aligned}
$$

We must verify that (4.21) is the necessary and sufficient condition for the vanishing of the right hand member of $(4.22)$. Let $\left(e_{j}\right)(1 \leqq j \leqq 6)$ be a canonical tangent frame at any point of $x$, i.e. a frame such that $\left(N, e_{1} d X, \cdots, e_{6} d X\right)$ is a canonical frame at the corresponding point for $Y$. In the sequel of this proof it is convenient to replace the frame $\left(\boldsymbol{e}_{j}\right)$ formally by the corresponding complex frame $\left(\boldsymbol{e}_{j}^{\prime}\right)$ defined by

$$
\boldsymbol{e}_{\alpha}^{\prime}=\frac{1}{2}\left(\boldsymbol{e}_{2 \alpha-1}-i \boldsymbol{e}_{2 \alpha}\right) ; \quad \mathbf{e}_{3+\alpha}^{\prime}=\left(\boldsymbol{e}_{\alpha}^{\prime}\right)^{-}=\frac{1}{2}\left(\boldsymbol{e}_{2 \alpha-1}+i \mathbf{e}_{2 \alpha}\right) \quad(\alpha=1,2,3)
$$

or by the inverse transformation

$$
e_{2 \alpha-1}=e_{\alpha}^{\prime}+e_{3+\alpha}^{\prime} ; \quad e_{2 \alpha}=i\left(e_{\alpha}^{\prime}-e_{3+\alpha}^{\prime}\right) .
$$

In terms of the new frame it is easy to express the formula for the inner product, the action of $J$, the triple scalar product as it appears in (4.22), and the property of any vector (or tensor) being real; we shall use Latin indices in the range from 1 to 6 , Greek indices from 1 to 3 , and starring an index to express addition or subtraction of 3 to its value.

A vector $\boldsymbol{u}=u^{i} \boldsymbol{e}_{j}^{\prime}$ (using the summation convention) is real, if and only if $u^{i *}=\bar{u}^{i}$; similarly a tensor is real, if and only if any two components related to each other by starring all the indices are mutually complex conjugates. The action of $J$ is described by $\boldsymbol{e}_{\alpha}^{\prime} J=i \boldsymbol{e}_{\alpha}^{\prime}, \boldsymbol{e}_{\alpha}^{\prime} \cdot J=-i \boldsymbol{e}_{\alpha}^{\prime} \cdot:$ the scalar product is derived from the identity $2 \boldsymbol{e}_{j}^{\prime} \cdot \boldsymbol{e}_{k}^{\prime}=\delta_{j k} \cdot$. Finally, if $\boldsymbol{u}=u^{i} \boldsymbol{e}_{j}^{\prime}, \boldsymbol{v}=v^{j} \boldsymbol{e}_{j}^{\prime}, \boldsymbol{w}=w^{j} \boldsymbol{e}_{j}^{\prime}$ are any three vectors, then the triple scalar product $(u d X, v d X, w d X)$ is given by

$$
(u d X, v d X, w d X)=\frac{1}{2}\left|\begin{array}{ccc}
u^{1} & u^{2} & u^{3} \\
v^{1} & v^{2} & v^{3} \\
w^{1} & w^{2} & w^{3}
\end{array}\right|+\frac{1}{2}\left|\begin{array}{ccc}
u^{4} & u^{5} & u^{6} \\
v^{4} & v^{5} & v^{6} \\
w^{4} & w^{5} & w^{6}
\end{array}\right| .
$$

Let the tensor components of $K$ be expressed by the $6 \times 6$ matrix $K_{j}^{k}$ : then the matrix satisfies the condition that $K$ is real, $K_{f^{*}}^{k^{*}}=\left(K_{j}^{k}\right)^{-}$, and symmetric, $K_{k}^{j}=K_{j^{*}}^{k^{*}}=\left(K_{j}^{k}\right)^{-}$. Hence the scalar $K_{\alpha}^{\alpha}$ satisfies the identity

$$
K_{\alpha}^{\alpha}=K_{\alpha^{*}}^{\alpha^{*}}=\frac{1}{2} K_{j}^{j}=3 H,
$$

where $H$ is the mean curvature.

From the above identities one can determine completely $d \omega$ by evaluating $d \omega\left(\boldsymbol{e}_{i}^{\prime} ; \boldsymbol{e}_{j}^{\prime} ; \boldsymbol{e}_{k}^{\prime}\right)$ for $i<j<k$ : 


$$
\begin{aligned}
& d \omega\left(\boldsymbol{e}_{1}^{\prime} ; \mathbf{e}_{2}^{\prime} ; e_{3}^{\prime}\right)=-K_{\alpha}^{\alpha}=-3 H ; \\
& d \omega\left(\boldsymbol{e}_{\alpha}^{\prime} ; e_{\beta}^{\prime} ; e_{\delta^{*}}^{\prime}\right)=-\epsilon_{\alpha \beta \gamma} K_{\delta^{*}}^{\gamma} \\
& \quad\left(\epsilon_{\alpha \beta \gamma}=\text { sign of of permutation of } 1,2,3 \text { to } \alpha, \beta, \gamma\right) ; \\
& d \omega\left(e_{\delta}^{\prime} ; e_{\alpha^{*}}^{\prime} ; e_{\beta^{*}}^{\prime}\right)=-\epsilon_{\alpha \beta \gamma} K_{\delta}^{\gamma^{*}} ; \\
& d \omega\left(e_{4}^{\prime} ; e_{5}^{\prime} ; e_{6}^{\prime}\right)=-K_{\alpha^{*}}^{\alpha^{*}}=-3 H,
\end{aligned}
$$

where the last two equations are the complex conjugates of the first two. If we compute the tensor components of $J K J$, we notice that they are equal or opposite to those of $K$, according as to whether the indices (written as Greek letters with or without star) are of opposite type (one starred, the other not) or of the same type respectively. Thus the linear operator $K+J K J$ has tensor components equal to $2 K$, if they are of opposite type, and zero, if they are of the same type. From these considerations and from the last set of equations it follows that (4.21) is necessary and sufficient in order that $d \omega=0$, completing the proof of the theorem.

The same argument as the one used in the Corollary to Theorem 4 leads to the analogous result for symplectic structures as follows.

CoROllary 1. If $x$ is a compact, differentiable manifold with the Hermitian, almost complex structure induced by a regular mapping $F$ of $X$ into $\mathcal{Y}$, then $X$ can not be a symplectic manifold.

Combining the results of Theorems 4 and 5 we obtain the criterion for an Hermitian, almost complex structure induced by $F$ to be Kählerian.

Corollary 2. An oriented, 6-dimensional manifold $x$ with the Hermitian, almost complex structure induced by a differentiable immersion $F$ of $x$ into $y$ is Kählerian, if and only if $F$ maps $X$ into a hyperplane.

Proof. From the remarks following Definition 3.4 it is clear that $x$ is Kählerian, if and only if it is simultaneously symplectic and integrable to a complex manifold, and hence if it satisfies simultaneously Equations (4.14) and (4.21). The two equations combined imply that $K$ must vanish identically, and therefore that $N$ is constant. Since $d(N \cdot X)=N \cdot d X=0$ for $N$ constant, it follows that $F$ satisfies the linear equation $X \cdot N=$ const. The converse of the argument being trivial, the statement is proved.

Having verified through the last corollary that $F$ induces a Kählerian structure on $x$ only in the trivial case where $F(x)$ is a hyperplane, it is natural to ask in which other cases, if any, the Hermitian almost complex structure induced on $x$ by $F$ is merely symplectic, or, alternatively, integrable to a complex analytic one: a complete solution of these two problems amounts to a complete characterization and functional representation of all hypersurfaces in $\mathcal{Y}$ that satisfy respectively (4.21) or (4.14), and is probably very 
difficult. As a matter of fact, for the first problem, we neither know as yet of any example, nor can show the impossibility of a hypersurface in $\mathcal{Y}$, other than a hyperplane, whose induced Hermitian structure is symplectic. On the other hand we shall show presently that there is a simple representation, albeit far from exhaustive as far as we can guess, of a large class of hypersurfaces in $\mathcal{Y}$, whose almost complex structure is integrable to a complex analytic one: these manifolds are closely related to the minimal surfaces in Euclidean 3-space.

Consider a linear subspace $\mathcal{Y}^{\prime}$ of $\mathcal{Y}$, closed under the operation of cross product $A \times B$, but in which the triple vector product $[A B C]$ vanishes identically. By Theorem $1 \mathcal{Y}^{\prime}$ is completely characterized as the linear subspace of $Y$ that is spanned by the first three vectors $I_{1}^{(0)}, I_{2}^{(0)}, I_{3}^{(0)}$ in some fixed, canonical frame for $\mathcal{Y},\left(I_{j}^{(0)}\right)(j=1,2, \cdots, 7)$; denote by $\mathcal{Y}^{\prime \prime}$ the orthogonal complement of $y^{\prime}$ in $\mathcal{Y}$, i.e. the oriented, linear subspace spanned by $\left(I_{j}^{(0)}\right)(j=4,5,6$, 7 ), and identify $\mathcal{Y}$, considered as a linear space, with the Cartesian product $\mathcal{Y}^{\prime} \times \mathcal{Y}^{\prime \prime}$.

Let $x^{\prime}$ be an oriented, differentiable (2-dimensional) surface and $F^{\prime}$ a regular, $\mathfrak{e}^{2}$ mapping of $\mathfrak{X}^{\prime}$ in to the oriented 3 -space $\mathcal{Y}^{\prime}$. Then one can naturally define from $F^{\prime}$ a regular mapping $F$ of the oriented, 6-dimensional manifold $x=X^{\prime} \times \mathcal{Y}^{\prime \prime}$ into $\mathcal{Y}=\mathcal{Y}^{\prime} \times \mathcal{Y}^{\prime \prime}$. as the product mapping of $F^{\prime}$ in $x^{\prime}$ with the identity in $Y^{\prime \prime}$. We shall determine the conditions on $F^{\prime}$ in order that the almost complex structure induced on $X$ by the product mapping $F$ be integrable to a complex analytic one.

THEOREM 6. Let $X$ be the product manifold $\boldsymbol{X}^{\prime} \times \mathcal{Y}^{\prime \prime}$ of an oriented surface $\mathfrak{X}^{\prime}$ with the 4-dimensional linear subspace $\mathcal{Y}^{\prime \prime}$ of $\mathcal{Y}$ that is spanned by the vectors $I_{4}^{(0)}, \cdots, I_{7}^{(0)}$ of a fixed, canonical frame $\left(I_{j}^{(0)}\right)(1 \leqq j \leqq 7)$ in $Y$. Let $F^{\prime}$ be any regular $\mathfrak{e}^{2}$ mapping of $\mathfrak{x}^{\prime}$ into the linear subspace $\mathcal{Y}^{\prime}$ of $\mathcal{Y}$ spanned by $I_{1}^{(0)}, I_{2}^{(0)}$, $I_{3}^{(0)}$. Then the product mapping $F$ of $X$ into $y$ defines an almost complex structure in $X$ that is integrable to a complex analytic one, if and only if $F^{\prime}$ maps $X^{\prime}$ locally as a minimal surface in the 3-space $y^{\prime}$.

Proof. Since the normal vector $N$ at each point $p \in X$ lies obviously in a linear 3-space parallel to $\mathcal{Y}^{\prime}$, if one starts to build a variable, canonical frame $\left(I_{j}\right)$ for $y$ at $X=F(p)$ by setting $I_{1}=N$ and $I_{2}=e_{1} d X$, where $e_{1}$ is a tangent vector in $X$ parallel to $X^{\prime}$, then $I_{3}=I_{1} \times I_{2}=e_{2} d X$ lies again in a 3 -space parallel to $\mathcal{Y}^{\prime}$, and hence $\boldsymbol{e}_{2}$ is also tangent to $\boldsymbol{X}^{\prime}$. One can then continue the construction of the local canonical frame $I_{j}=e_{j-1} d X(j=4,5,6,7)$, where these last four vectors come from $Y^{\prime \prime}$. It is also clear that the only contribution to the second fundamental form $u K \cdot v=u \cdot v K$ can come only from the 2-dimensional linear subspace of the tangent space to $X$ coming from $x^{\prime}$. Since $\left(\boldsymbol{e}_{j}\right)$ is a unitary frame with respect to the Hermitian, almost complex structure of $x$, we can write the second fundamental form in matrix form as follows 


$$
\begin{aligned}
& \boldsymbol{e}_{j} K=a_{j 1} \mathbf{e}_{1}+a_{j 2} \mathbf{e}_{2} \\
& \boldsymbol{e}_{j} K=0
\end{aligned}
$$

$$
\begin{array}{r}
(j=1,2), \\
(j=3,4,5,6),
\end{array}
$$

where

$$
a_{12}=a_{21} .
$$

Now, since $e_{j} J=-(-1)^{i} \boldsymbol{e}_{j^{\prime}}$, where $j^{\prime}=j-(-1)^{i}$, we can write directly the formulas for $e_{j}(K-J K J)$ whose vanishing is equivalent to the integrability condition (4.14) of Theorem 4:

$$
\begin{array}{lr}
\boldsymbol{e}_{j}(K-J K J)=\left(a_{11}+a_{22}\right) \boldsymbol{e}_{j} & (j=1,2), \\
\boldsymbol{e}_{j}(K-J K J)=0 & (j=3,4,5,6) .
\end{array}
$$

Thus we have proved that the almost complex structure induced on $x$ by $F$ is integrable, if and only if

$$
a_{11}+a_{22}=0 .
$$

But since the matrix $\left(a_{j k}\right)(j, k=1,2)$ clearly represents the second fundamental form for $X^{\prime}$ under $F^{\prime}$ in terms of the tangent frame $e_{1}, e_{2}$, the trace $a_{11}+a_{22}$ is twice the mean curvature of $F^{\prime}\left(X^{\prime}\right)$ in $Y^{\prime}$. This concludes the proof of the theorem.

It is important to bear in mind that the almost complex structure induced on $X$ by $F$ is not the product structure, except in the trivial case where $F^{\prime}\left(\mathscr{X}^{\prime}\right)$ lies in a plane. The reason for this is that, although for each point $\boldsymbol{p}^{\prime} \in \mathfrak{X}^{\prime}$ the submanifold $\left\{\boldsymbol{p}^{\prime}\right\} \times \mathcal{Y}^{\prime \prime}$ of $\boldsymbol{X}$ is a complex analytic submanifold of $x$ (even when the integrability condition in the latter is not satisfied) isomorphic to the space of 2 complex variables, as $p^{\prime}$ varies over $x^{\prime}$ the corresponding translation of $F\left(\left\{\boldsymbol{p}^{\prime}\right\} \times \mathcal{Y}^{\prime \prime}\right)$ in $\mathcal{Y}$ is not complex analytic, owing to the fact that the normal vector $N$ (which alone determines the complex structure in the tangent space) is variable.

We shall prove now one last general property of the Hermitian, almost complex structure induced on any 6-dimensional manifold $x$ by a regular mapping $F$ of $X$ into $\mathcal{Y}$, namely that (cf. Definition 3.3) $X$ becomes a special Hermitian manifold in all cases.

THEOREM 7. Given an oriented, differentiable, 6-dimensional manifold $\mathcal{X}$ and a regular, differentiable mapping $F$ of $X$ into the Cayley space $Y$, then $F$ induces on $X$ the structure of a special Hermitian (almost complex) manifold.

Proof. We shall consider as allowable local tangent frames in the neighborhood of any point in $X$ any sextuple $\left(\boldsymbol{e}_{j}\right)$ of tangent vector functions such that the septuple $\left(N, e_{1} d X, \cdots, e_{6} d X\right)$ at the image point $X$ is a canonical frame for $Y$. Then the frame $\left(e_{j}\right)$ is unitary with respect to the Hermitian, almost complex structure induced on $X$. The statement of Theorem 2 is that any two overlapping tangent frames $\left(I_{j}\right)\left(I_{j}=\boldsymbol{e}_{j-1} d X, 2 \leqq j \leqq 7\right)$ are related to one 
another by a linear transformation $\sigma$, which, since $I_{1}=N$ is invariant, under the representation $\phi$ defined by (2.4) is representable in a unique way by a matrix $\phi(\sigma) \in S U(3)$. The corresponding frame transformation $\tilde{\sigma}$ of one system $\left(\boldsymbol{e}_{j}\right)$ to another can be described uniquely by a unitary, $3 \times 3$ matrix $\Theta=\left(\eta_{\beta}^{\alpha}+i \theta_{\beta}^{\alpha}\right)$ as in $\left(3.6^{\prime}\right)$ under the representation $\psi$ of the tangent space in $x$ defined by (3.4). From the definitions of the representations $\phi$ and $\psi$ of vectors respectively at a point $p \in X$ and in the orthogonal complement of $N(p)$ in $\mathcal{Y}$ we obtain the identity for corresponding frames $\left(\boldsymbol{e}_{j}\right)$ and $\left(N, \boldsymbol{e}_{j} d X\right)$

$$
u^{\psi}=(u d X)^{\phi},
$$

and hence the transformations of allowable frames satisfy the relation

$$
\psi(\tilde{\sigma})=\Theta=\phi(\sigma) \in S U(3) .
$$

Hence the manifold $X$ with the covering by allowable, local tangent frames is a special Hermitian manifold, as we set out to prove. In other words the first Chern characteristic class $c^{1}$ of every almost complex manifold obtained by our method vanishes.

5. Examples of compact, complex analytic manifolds. We shall apply now the results of $\S 4$ to construct some compact, complex analytic manifolds with 3 complex dimensions, that exhibit some interesting properties.

Let $X^{\prime}$ be a closed, hyperelliptic Riemann surface of odd genus $g=2 h+1$ $(h \geqq 1)$. Without loss of generality $x^{\prime}$ can be considered as the algebraic curve defined by the equation in the complex field

$$
w^{2}=f_{k}(z),
$$

where $f_{k}(z)$ is an arbitrary polynomial of degree $k=2 g+2=4 h+4$, with no multiple roots. The Abelian differentials of the first kind in $X^{\prime}$ are then given by

$$
\omega=\phi(z) w^{-1} d z,
$$

where $\phi(z)$ is an arbitrary polynomial of degree at most $g-1=2 h$ : we shall single out one such polynomial $\phi(z)$ of degree equal to $h$ and in terms of it take the following three differentials of the first kind,

$$
\begin{aligned}
& \omega_{1}=\frac{1}{2}\left(\phi^{2}(z)-1\right) w^{-1} d z, \\
& \omega_{2}=\phi(z) w^{-1} d z, \\
& \omega_{3}=\frac{1}{2 i}\left(\phi^{2}(z)+1\right) w^{-1} d z .
\end{aligned}
$$

The above construction was made in order to exhibit a closed Riemann surface $X^{\prime}$ with three Abelian differentials of the first kind $\omega_{j}(j=1,2,3)$ with the following properties: 
(a) linear independence;

(b) the three differentials satisfy the quadratic equation

$$
\omega_{1}^{2}+\omega_{2}^{2}+\omega_{3}^{2}=0
$$

(c) there is no place (point) in $X^{\prime}$ where the three differentials $\omega_{j}$ vanish simultaneously.

It is clear that the three differentials chosen satisfy properties (a) and (b); to verify property (c) we point out that it is obvious that $\omega_{1}$ and $\omega_{3}$ can not vanish simultaneously at any place where $z$ is finite, so that there remains only to check the two places where $z$ becomes infinite: at each of those places $d z$ has a pole of order $2, w^{-1}$ has a zero of order $2 h+2$, and $\phi^{2}(z)$ has a pole of order $2 h$; hence neither $\omega_{1}$ nor $\omega_{3}$ vanishes at either of the two places where $z$ is infinite, completing the argument.

Consider now the three real-valued functions of a place $p^{\prime} \in X^{\prime}$

$$
x^{j}\left(\boldsymbol{p}^{\prime}\right)=\operatorname{Re} \int_{p_{0}^{\prime}}^{p^{\prime}} \omega_{j} \quad(j=1,2,3)
$$

as defining for any fixed place $\boldsymbol{p}_{0}^{\prime} \in \mathfrak{X}^{\prime}$ a real analytic mapping of the maximal Abelian covering $\widetilde{\boldsymbol{X}}^{\prime}$ of $\boldsymbol{X}^{\prime}$ into the Euclidean 3-space with Cartesian coordinates $x^{j}$. This mapping is regular, since the differentials $\omega_{j}$ satisfy property (c) (see above); by a classical theorem attributed to Monge, Lie, and Weierstrass (cf. $[24$, p. 184]) property (b) is equivalent to the statement that the functions $x^{j}\left(\tilde{\boldsymbol{p}}^{\prime}\right)$ (where $\tilde{\boldsymbol{p}}^{\prime}$ is a point of $\widetilde{\boldsymbol{X}}^{\prime}$ covering $\boldsymbol{p}^{\prime} \in \boldsymbol{X}^{\prime}$ ) map $\tilde{\boldsymbol{X}}^{\prime}$ locally into a minimal surface (globally the image is, in general, everywhere dense); finally, because of property (a), it follows that $x^{\prime}$ is not mapped into a plane. This method of generating minimal surfaces was studied extensively by $\mathrm{H}$. A. Schwarz [22] in connection with the Plateau problem. We remark in addition that the covering transformations of the image of $\tilde{\mathfrak{x}}^{\prime}$ in the Euclidean 3space over the original Riemann surface $x^{\prime}$ are given by translations of the space, corresponding to the real parts of the periods of $\int \omega_{j}$.

Let $\tilde{x}$ be the product manifold $\tilde{\mathbb{X}}^{\prime} \times \mathcal{Y}^{\prime \prime}$ of the maximal Abelian covering $\tilde{x}^{\prime}$ of $x^{\prime}$ with the real linear, 4-dimensional subspace $\mathcal{Y}^{\prime \prime}$ of $\mathcal{Y}$ that is spanned by the last four vectors of a fixed, canonical frame $\left(I_{j}^{(0)}\right)$; let $F^{\prime}$ be the regular mapping of $\tilde{\mathfrak{X}}^{\prime}$ into the 3 -dimensional subspace $\mathcal{Y}^{\prime}$ of $\mathcal{Y}$ that is spanned by the first three vectors, defined by

$$
F^{\prime}\left(\tilde{\boldsymbol{p}}^{\prime}\right)=\sum_{j=1}^{3} x^{j}\left(\tilde{\boldsymbol{p}}^{\prime}\right) I_{j}^{(0)},
$$

where $x^{i}\left(\tilde{\boldsymbol{p}}^{\prime}\right)$ is defined from the corresponding, multiple valued function on $x^{\prime}$ given by (5.4), and let $F$ be the product mapping of $F^{\prime}$ in $\tilde{x}^{\prime}$ with the identity mapping in $\mathcal{Y}^{\prime \prime}$, as in Theorem 6. Since $F^{\prime}$ maps $\tilde{\mathfrak{X}}^{\prime}$ locally into a minimal surface in $\mathcal{Y}^{\prime}$, the almost complex structure induced by $F$ on $\widetilde{\mathscr{X}}$ is 
integrable to a complex analytic one.

I.et $T^{4}$ denote a 4-dimensional torus obtained by factoring from $\mathcal{Y}^{\prime \prime}$ a free module generated by any four linearly independent vectors in $\mathcal{Y}^{\prime \prime}$, and let $X$ be the product manifold $x^{\prime} \times T^{4}$. Then $x$ is a compact, oriented, 6-dimensional manifold, whose maximal Abelian covering is $\widetilde{x}=\widetilde{x}^{\prime} \times \mathcal{Y}^{\prime \prime}$. To every covering transformation of $\widetilde{x}$ with respect to $x$ there corresponds a transformation of $F(\widetilde{X})$ onto itself that comes from a translation in $\mathcal{Y}$. Hence the covering transformations of $\tilde{\mathscr{X}}$ preserve the complex analytic structure, as well as the Hermitian metric and the allowable tangent frames (cf. Theorem 7 ); thus we arrive at the result that $F$ induces on the compact manifold $X$ the structure of a special Hermitian, complex analytic manifold. We shall now prove that $x$ does not admit a Kähler metric. In fact, if $x$ did admit a Kähler metric, then, since the first Betti number $B_{1}$ of $X$ is $2 g+4$ (because $B_{1}\left(X^{\prime}\right)=2 g$ and $B_{1}\left(T^{4}\right)=4$ ), by Hodge's theorem (cf. [14, p. 200]) there would exist $B_{1} / 2=g+2$ linearly independent, holomorphic, closed differential forms on $x$, which together with their complex conjugates would span linearly (with complex coefficients) the whole, $B_{1}$-dimensional space of harmonic 1 -forms on $X$; of these $g+2$ holomorphic forms $g$ at least are known, namely the injection in $x$ of the $g$ Abelian differentials of the first kind in $x^{\prime}$, say $\omega_{1}, \cdots, \omega_{0}$; since these differentials vanish identically when restricted to any toral submanifold $\left\{\boldsymbol{p}^{\prime}\right\} \times \mathcal{Y}^{\prime \prime}$ (for each $\boldsymbol{p}^{\prime} \in \mathcal{X}^{\prime}$ this is a complex analytic submanifold of $x$ ), if $x$ admitted a Kähler metric, there would be two more linearly independent, holomorphic, closed 1-forms, say $\omega^{\prime}, \omega^{\prime \prime}$, with nonzero integrals along the cyles lying in $\left\{\boldsymbol{p}^{\prime}\right\} \times \mathcal{Y}^{\prime \prime}$. From the multiplicative structure of the cohomology ring of $X$ it follows that the exterior 6 -form $\Psi$ defined by

$$
\Psi=-i \omega_{1} \wedge \bar{\omega}_{1} \wedge \omega^{\prime} \wedge \bar{\omega}^{\prime} \wedge \omega^{\prime \prime} \wedge \bar{\omega}^{\prime \prime}
$$

has a strictly positive integral over $x$, from which we deduce that the holomorphic 3-form $\Omega$ defined by

$$
\Omega=\omega_{1} \wedge \omega^{\prime} \wedge \omega^{\prime \prime}
$$

is not identically zero. Since $X$ has the structure of a special Hermitian manifold, the first Chern class $\mathrm{c}^{1}$ is zero, and hence the divisor of $\Omega$ must be homologous to zero. But the divisor of $\Omega$ is a union of complex analytic submanifolds counted with positive multiplicity, since $\Omega$ is holomorphic, and it is known (cf. [17]) that a cycle of this type can not be homologous to zero in a Kähler manifold unless it is itself zero. Hence the holomorphic 3-form $\Omega$ has no zeros. On the other hand the Abelian differential $\omega_{1}$ appearing as a factor of $\Omega$ in (5.7) certainly has some zeros (precisely of total multiplicity $2 g-2$ in $X^{\prime}$ ), so that the divisor of $\Omega$ in $X$ is nonempty, whence our assumption that $x$ admits a Kähler metric leads to a contradiction.

Consider now a comparison between the manifold $x$ and the product com- 
plex manifold $x^{\prime} \times T^{4}$ of the Riemann surface $x^{\prime}$ with any complex analytic manifold $T^{4}$ homeomorphic to a torus (e.g. by factoring out of the vector space of ordered pairs of complex numbers a free module generated by any four linearly independent translations). Then, since both $\mathscr{X}^{\prime}$ and $T^{4}$ admit a Kähler metric, so does the product manifold $x^{\prime} \times T^{4}$. Furthermore, since the canonical divisor of $x^{\prime}$ (see the discussion following Definition 3.3) is homologous to a point $p^{\prime} \in X^{\prime}$ counted with multiplicity $2 g-2$, and since the canonical divisor of $T^{4}$ is zero, it follows that the canonical divisor of $x^{\prime} \times T^{4}$ is homologous to $(2 g-2)\left(\left\{\boldsymbol{p}^{\prime}\right\} \times T^{4}\right)$. The two properties just described that distinguish the two complex manifolds $X$ and $x^{\prime} \times T^{4}$ from each other furnish the first known examples of compact, complex manifolds that are differentiably homeomorphic (preserving the natural orientation induced by the complex analytic structure), but whose Chern characteristic classes are distinct, and such that one, but not the other, manifold admits a Kähler metric. Examples of differentiably homeomorphic manifolds with almost complex structures inducing different first Chern classes were known previously, but, the examples given here being complex analytic, we can give a negative answer to a question raised by Hirzebruch [13, pp. 222-224 and 233], whether the Chern classes of a complex manifold depend on the differentiable structure and the orientation alone, and not on the particular complex analytic structure. The previous examples by Hirzebruch [12], of complex manifolds that are differentiably homeomorphic, but not complex analytically isomorphic had all the same Chern classes, and, since they could all be realized as algebraic manifolds, they all admitted Kähler metrics. We can thus state our final result:

If a compact, oriented, differentiable manifold admits more than one complex analytic structure compatible with the differentiable structure and the orientation, then the Chern classes are not necessarily uniquely determined in the manifold, nor is the property invariant, that a complex analytic structure in the manifold admits a Kähler metric.

In conclusion it seems likely that the following conjecture may be true, at least in the cases when the Chern classes are different from zero: If $X$ is a compact, complex manifold and $F$ a differentiable homeomorphism (in the real sense) of $X$ onto an algebraic manifold $x^{\prime}$ such that the Chern classes and the orientation are preserved, then does $X$ admit a Kähler metric?

\section{REFERENCES}

1. E. Calabi and D. C. Spencer, Completely integrable, almost complex manifolds, Bull. Amer. Math. Soc. Abstract 57-3-251.

2. E. Calabi and B. Eckmann, A class of compact, complex manifolds which are not algebraic, Ann. of Math. vol. 58 (1953) pp. 494-500.

3. E. Cartan, Groupes réels, finis et continus, Ann. École Norm. vol. 31 (1914) pp. 263-355; also: Oeuvres complètes, Partie I, vol. 1, Paris, Gauthier-Villars, 1952, pp. 399-491.

4. S. S. Chern, Characteristic classes in Hermitian manifolds, Ann. of Math. vol. 47 (1946) pp. 85-121. 
5. - Topics in differential geometry, mimeographed notes, Institute for Advanced Study, Princeton, 1951.

6. C. Chevalley, Theory of Lie groups I, Princeton University Press, 1946.

7. B. Eckmann and A. Frölicher, Sur l'intégrabilité des structures presque-complexes, C. R. Acad. Sci. Paris vol. 232 (1951) pp. 2284-2286.

8. C. Ehresmann, Sur les variétés presque-complexes, Proceedings of the International Congress of Mathematicians, 1950, vol. II, Amer. Math. Society, 1952, pp. 412-419.

9. C. Ehresmann and P. Libermann, Sur les structures presque hermitiennes isotropes, C. R. Acad. Sci. Paris vol. 232 (1951) pp. 1281-1283.

10. L. P. Eisenhart, Riemannian geometry, Princeton University Press, 1949.

11. H. Freudenthal, Oktaven, Ausnahmengruppen und Oktavengeometrie, Utrecht, 1951.

12. F. Hirzebruch, Über eine Klasse von einfach-zusammenhängenden komplexen Mannigfaltigkeiten, Math. Ann. vol. 124 (1951) pp. 77-86.

13. - Problems on differentiable and complex manifolds, Ann. of Math. vol. 60 (1954) pp. $213-236$.

14. W. V. D. Hodge, The theory and applications of harmonic integrals, Cambridge University Press, 1941.

15. E. Hopf, Über den funktionalen, insbesondere den analytischen Charakter der Lösungen elliptischer Differentialgleichungen zweiter Ordnung, Math. Zeit. vol. 34 (1932) pp. 194-233.

16. H. Hopf, Zur Topologie der komplexen Mannigfaltigkeiten, Studies and Essays presented to R. Courant, New York, 1948, pp. 167-185.

17. A. Lichnérowicz, Généralisations de la géométrie kählérienne globale, Colloque de géométrie differentielle, Louvain, 1951, pp. 99-122.

18. C. B. Morrey, Second order elliptic systems of differential equations, Contributions to the theory of partial differential equations, Ann. of Math. Studies, no. 33, Princeton University Press, 1954 , pp. 101-159.

19. R. Moufang, Zur Struktur von Alternativkörpern, Math. Ann. vol. 110 (1935) pp. 416430.

20. L. Nirenberg, On a generalization of quasi-conformal mappings and its application to elliptic partial differential equations, Contributions to the theory of partial differential equations, Ann. of Math. Studies, no. 33, Princeton University Press, 1954, pp. 95-100.

21. G. de Rham, Variêtés différentiables, Paris, Hermann, 1955.

22. H. A. Schwarz, Gesammelte mathematische Abhandlungen, vol. I, Berlin, Springer, 1890.

23. N. Steenrod, The topology of fibre bundles, Princeton University Press, 1951.

24. D. J. Struik, Differential geometry, Cambridge, Addison-Wesley, 1950.

25. K. Yano, Some remarks on almost complex manifolds, Proceedings of the International Congress of Mathematicians, 1954, vol. II, p. 268.

26. M. Zorn, Theorie der alternativen Ringe, Abh. Math. Sem. Hamb. Univ. vol. 8 (1931) pp. 123-147.

27. - Alternativkörper und quadratische Systeme, Abh. Math. Sem. Hamb. Univ. vol. 9 (1933) pp. 395-402.

\section{University of Minnesota, MinNeAPOLIS, MinN.}

